\title{
Perspective
}

PERSPECTIVE Actualité en histoire de l'art

1 | 2018

Actualité en histoire de l'art

\section{Les collections muséales d'art « non-occidental » : constitution et restitution aujourd'hui}

Un débat entre Viola König, Benoît de L'Estoile, Paula López Caballero, Vincent Négri, Ariane Perrin et Laurella Rinçon, conduit par Claire BoscTiessé

Viola König, Benoît de L'Estoile, Paula López Caballero, Vincent Négri, Ariane Perrin, Laurella Rinçon et Claire Bosc-Tiessé

\section{(2) OpenEdition}

Édition électronique

URL : http://journals.openedition.org/perspective/9059

DOI : 10.4000/perspective.9059

ISSN : 2269-7721

Éditeur

Institut national d'histoire de l'art

\section{Édition imprimée}

Date de publication : 30 juin 2018

Pagination : $37-70$

ISBN : 978-2-917902-46-2

ISSN : $1777-7852$

Référence électronique

Viola König, Benoît de L'Estoile, Paula López Caballero, Vincent Négri, Ariane Perrin, Laurella Rinçon et Claire Bosc-Tiessé, « Les collections muséales d'art « non-occidental » : constitution et restitution aujourd'hui », Perspective [En ligne], 1 | 2018, mis en ligne le 28 novembre 2018, consulté le 10 décembre 2020. URL : http://journals.openedition.org/perspective/9059 ; DOI : https://doi.org/ 10.4000/perspective.9059 


\title{
Les collections muséales d'art « non-occidental » : constitution et restitution aujourd'hui
}

\author{
Un débat entre Viola König, Benoît de L'Estoile, \\ Paula López Caballero, Vincent Négri, Ariane Perrin \\ et Laurella Rinçon, conduit par Claire Bosc-Tiessé
}

Dans l'histoire des objets, le passage d'un lieu d'usage actif au musée, intrinsèquement lié à la conservation et à la recomposition des identités et des nations, a souvent constitué une rupture violente, d'autant plus lorsque ce passage a été imposé de l'extérieur et lorsqu'il coïncide avec un transfert au loin, dans un contexte de domination qu'il soit politique, militaire ou économique. Pour ce qui concerne l'Afrique, Ezio Bassani a montré le peu d'objets, identifiés comme venant d'Afrique et conservés, entrés dans les collections occidentales avant 1800 : environ cinq cent cinquante pour quinze pays européens plus les États-Unis. Ils sont plus d'une centaine de milliers (plusieurs centaines?) aujourd'hui. Il s'agit d'objets de toute sorte, objets de culte, objets de prestige ou de la vie quotidienne, armes et instruments de musique ${ }^{1}$. Même si ces chiffres restent à préciser, les rappeler donne du corps à l'ampleur de ce qui s'est déroulé au cours des XIXe et $X X^{e}$ siècles. On connaît - bien relativement - certains butins de guerre, quelques grandes expéditions de recherche et d'acquisition de la première moitié $d u X X^{e}$ siècle, le pillage archéologique qui a pris une ampleur sans précédent dans les années 1990, le reste peu. L'histoire des objets que les institutions muséales rangent dans une catégorie non-occidentale n'est toutefois pas bipolaire : le moment où ils sont hors Occident, le moment où ils sont en Occident. Ils ont souvent passés par plusieurs phases de transformation de leurs usages et de leurs statuts, dans la société d'origine qui les a produits comme dans tous les endroits où ils ont été emportés, de gré ou de force; ils ont parfois connu des périodes d'abandon, voire d'oubli, puis de redécouverte, et en tout cas de réinvestissement, par les descendants de ceux qui les avaient produits, ou par d'autres étrangers à leur production initiale. Par ailleurs, les objets dotés d'une charge symbolique forte, ou importants pour un pays ou un groupe de personnes à un titre ou à un autre, ne sont pas nécessairement des objets produits par eux ou pour eux. Il faudra savoir prendre en compte cette histoire longue dans toute sa complexité.

Lorsque nous avons proposé aux contributeurs réunis ici de participer à ce débat, le discours de Ouagadougou n'avait pas encore été prononcé. Ce sujet sur la restitution était sur la table du comité de rédaction de Perspective, comme un serpent de mer de l'histoire de l'art et des études muséographiques, voire comme un marronnier ${ }^{2}$. Il y avait bien la demande de restitution déposée par le Bénin en 2016, le refus de la France. Mais les jeux semblaient faits, entre demande, refus ou esquive; pas vraiment de nouvelle donne dans le domaine politique en France. Depuis plus d'une vingtaine d'années, les conditions d'acquisition, leurs implications dans les relations internationales, les systèmes économiques, la construction des identités et des États, les ruptures de transmission et les réappropriations symboliques s'étaient toutefois inscrits au programme

Chercheure au CNRS, Claire

Bosc-Tiessé est conseillère scientifique à I'INHA, responsable du domaine " Histoire de l'art du XIVe au XIX ${ }^{\mathrm{e}}$ siècle " et du programme " Vestiges, indices, paradigmes : lieux et temps des objets d'Afrique, XIVe-XIXe siècles ». Elle mène plusieurs projets de recherche collectifs sur l'Éthiopie entre le XIII et le XVIII ${ }^{e}$ siècle.

Viola König a été la directrice de l'Ethnologisches Museum de Berlin (2001-2017) et du

Übersee-Museum Bremen (1992-2001). Elle est professeure en Sciences culturelles à l'Universität Bremen et en Études précolombiennes à la Freie Universität Berlin. Elle a été chargée de la conception de la muséographie du Humboldt Forum à Berlin.

Benoît de L'Estoile, anthropologue, est directeur de recherche au CNRS (Centre

Maurice Halbwachs) et professeur à I'École normale supérieure (PSL). II travaille notamment sur les héritages coloniaux dans les musées et le patrimoine ; il est l'auteur de Le goût des Autres. De l'exposition coloniale aux Arts premiers (Flammarion, 2007). 
Historienne et anthropologue, Paula López Caballero est chercheure au CNRS (Sciences Po - CERI) et à I'Universidad Nacional Autónoma de México (CEIICH-UNAM). Son travail porte sur les processus historiques et les pratiques sociales contemporaines conditionnant I'organisation de la distinction entre " Indien » et « non-Indien » au Mexique, comprise comme un aspect des trajectoires historiques de formation de

l'État et la nation. Sa dernière publication est un ouvrage collectif intitulé Beyond Alterity. Destabilizing the Indigenous Subject in Mexico.

Vincent Négri est chercheur à I'Institut des Sciences sociales du Politique (UMR 7220 CNRS, ENS Paris-Saclay, université Paris Nanterre). Ses travaux de recherche et ses publications portent sur le droit comparé et le droit international de la culture et du patrimoine culturel, ainsi que sur les rapports entre normes et cultures.

Ariane Perrin est historienne de I'art et spécialiste de I'histoire de l'art de la Corée qu'elle enseigne à I'Institut catholique de Paris. Elle est chercheure associé à I'UMR 8173 "Chine, Corée, Japon » CNRS - EHESS et travaille notamment sur la question des restitutions des biens culturels coréens exportés illégalement.

Laurella Rinçon est conservatrice du patrimoine, spécialiste des arts d'Afrique. Elle est experteassociée à la Division du patrimoine de l'UNESCO et chercheure-associée au département des Sciences sociales de I'École normale supérieure de Paris, où elle collabore

à I'axe "Musées et héritages coloniaux ». Elle étudie les processus de créolisation dans les arts d'Afrique et la mutation des musées européens d'ethnographie, en particulier en Suède, aux Pays-Bas et en France. des séminaires de recherche, voire de certains musées ou à l'initiative d'associations, afin de réfléchir au sens de ces objets pour les descendants des populations dont ils proviennent, quand il est possible de tracer une filiation. Il paraissait donc important de faire le point sur l'avancée des recherches, menées trop souvent dans l'ombre en dépit de la position étatique, les questions concernant les collections publiques françaises se heurtant de toute manière au mur de l'inaliénabilité des collections. Parmi les gestes présidentiels des années 1990 et 2000 demeuraient dans les mémoires la gestion de la restitution des manuscrits coréens par Jacques Chirac, puis Nicolas Sarkozy ; la négociation autour des sculptures Nok, sorties illégalement du Nigéria, achetées sur le marché de l'art en prévision de l'ouverture du musée du Quai Branly, rendues légalement au Nigéria mais conservées en dépôt en France et présentées au Pavillon des Sessions du Louvre 3 . Dans un geste où l'on ne sait ce qui l'emporte du politique ou du cynique, Jacques Chirac avait de même retourné, en 1998, une sculpture en terre cuite provenant de fouilles clandestines dans le delta intérieur du Niger au Mali, qui avait été acquise sur le marché de l'art et lui avait été offerte par des amis. Elle est aujourd'hui présentée au musée de Bamako avec la mention "don de Jacques Chirac, président de la République française ${ }^{4}$ ". Dans ce contexte, Emmanuel Macron, exprimant à l'université de Ouagadougou, au Burkina Faso, le 28 novembre 2017, la volonté qu'au cours de son mandat "les conditions soient réunies pour des restitutions temporaires ou définitives du patrimoine africain en Afrique», crée une rupture, au moins dans le discours.

Ce débat ne concerne toutefois pas seulement les objets africains ni les relations de la France avec ses anciennes colonies. Il s'agit au contraire de multiplier et de combiner les focales pour comparer les cas de figure à différentes échelles par le biais du droit, de l'anthropologie, des sciences politiques ou de l'histoire de l'art, par des chercheurs qui ont parfois cumulé leurs travaux avec la gestion de collections dans différents pays. On verra que le débat est loin d'être clos, espérons qu'il donnera quelques pistes pour mieux comprendre les enjeux, voire décaler les questions et poser les bases d'un regard réflexif sur les différents positionnements ${ }^{6}$. [Claire Bosc-Tiessé]

Claire Bosc-Tiessé. La notion de collections extra ou non-occidentales est une catégorie qui ne fait pas sens pour l'historien, sauf à l'étudier en elle-même, mais c'est une catégorie hétéroclite qui est utilisée dans le monde des musées pour rassembler sous un même chapeau des objets de provenances diverses. L'emploi de cette catégorie est aussi varié, incluant diversement les objets asiatiques, comprenant ou non les objets égyptiens d'époque pharaonique, pour ne donner que ces deux exemples. Ce n'est toutefois pas seulement une catégorie pragmatique, elle reflète aussi fondamentalement un positionnement, au moins de fait, par rapport au monde. Eu égard à celui-ci, nous posons donc la question de la restitution des collections non-occidentales. Mais cela fait-il sens d'une manière ou d'une autre ? Le dénominateur commun est-il l'acquisition ou l'appropriation en situation de domination quelle qu'elle soit? Peut-on par exemple penser et traiter de la même manière les frises du Parthénon et les objets du royaume de Bénin ou les butins militaires et les pièces archéologiques issues de fouilles clandestines?

Laurella Rinçon. Les objets collectés en Afrique, dans les Amériques, en Asie, et en Océanie ont, depuis le XVle siècle, connus des désignations multiples. Des curios exotiques des cabinets de curiosité aux objets ethnographiques, en passant par les fétiches, l'art nègre, l'art primitif, les arts lointains, tribaux, exotiques, primordiaux ou premiers, et enfin collections " extra » ou "non-occidentales», ces tâtonnements terminologiques dépeignent surtout le besoin de l'Occident d'inventer un " Autre » et de circonscrire un « Ailleurs » qu'il découvre et qu'il tente de maîtriser. Il s'agit donc 
d'une multitude de corpus, réunis sous un vocable générique niant la complexité des contextes de création, afin d'appréhender leur diversité sous une seule et englobante appellation, laquelle s'avère nécessairement imprécise. Au-delà du contexte d'acquisition ou d'appropriation, c'est réellement la construction d'un rapport à l'autre qui s'opère dans cette partition où l'Occident se distingue du reste du monde.

Cette multiplicité de termes se répercute naturellement sur le processus de dénomination des institutions qui abritent de telles collections. Ces vingt dernières années, les musées d'ethnographie se sont transformés dans toute l'Europe en « musées des cultures du monde ", à Leyde, à Göteborg, ou à Vienne, après avoir connu les mêmes hésitations sur l'intitulé le plus approprié. On est ainsi passé, à Paris, du musée des Arts premiers, au musée des Arts et Civilisations puis, comme par défaut, au musée du quai Branly.

C'est cette vision du monde centrée sur l'Occident que la plupart des institutions perpétuent jusque dans leurs projets de refondation aujourd'hui, à l'exception du Musée d'ethnographie de Genève qui présente des collections européennes dans son exposition permanente. De ce point de vue, la constitution de ces collections continue de répondre à la même logique hier et aujourd'hui, alors que les demandes de restitutions qu'elles motivent, en revanche, ne sont pas dissociées des enjeux et problématiques liées plus globalement à ce débat. En effet, par le biais de la question des restitutions, ces objets sont examinés dans leur singularité, car c'est l'histoire du parcours d'un objet qui détermine les conditions de sa restitution au pays d'origine. Le débat sur les restitutions invite donc à un décloisonnement de ces catégories surimposées et muettes, et à considérer l'unicité de chaque objet.

Benoît de L'Estoile. La catégorie " extra-occidental » est une notion pseudogéographique, qui se présente comme factuelle, mettant l'accent sur la provenance des objets. Elle repose sur une définition négative : ce qui n'est pas occidental, c'est-à-dire ce qui ne provient pas de "chez nous », mais a été approprié comme faisant partie de «notre » patrimoine. Elle correspond précisément à ce que j'appelle le « musée des Autres », construit comme un miroir du «musée du Nous ».

La notion renvoie à l'histoire de la constitution des catégories, elle-même insérée dans l'histoire des relations entre l'Europe et les autres continents. Ainsi cette catégorie, comme nombre d'autres, figure au nombre de ce que j'appelle héritage colonial. La plupart des objets aujourd'hui présents dans les collections de musées « occidentaux » n'ont pas été acquis en tant que « butin » (de guerre, de pillage), mais dans une configuration marquée par un « rapport colonial ${ }^{8} »$. C'est cette dimension relationnelle, que masque la catégorie " extra-occidental », qu'il s'agit d'éclairer, en insistant sur le parcours plus que sur l'origine. Typiquement, un cartel d'objet donne les matériaux de l'objet, son origine ethno-géographique, et éventuellement le nom des collectionneurs (occidentaux) entre les mains desquels il est passé, élidant ainsi toute l'histoire du parcours par lequel l'objet est parvenu jusqu'à la vitrine du musée européen où le visiteur peut le contempler.

L'histoire des objets et des collections apparaît dès lors pour les musées non plus comme une activité annexe (de type historique et mémoriel), mais centrale du point de vue des héritages coloniaux99. Il s'agit donc de poser systématiquement un certain nombre de questions : comment les objets sont-ils arrivés au musée ? Dans quelles circonstances? Quel est leur statut juridique? Y-a-t-il des personnes (individuelles ou collectives) qui revendiquent les objets ou bien se revendiquent de ceux-ci - c'est-àdire qui revendiquent un lien privilégié avec des choses appartenant aux collections du musée : des objets, des restes humains, des photographies, des enregistrements de sons ou de paroles, etc. ? L'importance de retracer cette histoire des relations n'est pas seulement d'ordre historique, mais prend aussi sens par rapport au présent. 
Paula López Caballero. Réfléchir au périmètre de la catégorie de collections « extra- » ou «non occidentales » ouvre la voie à un questionnement d'anthropologie politique, pouvant compléter les perspectives de l'histoire de l'art ou de la conservation du patrimoine. En effet, comprendre comment prend forme la communauté politique à quelque niveau que ce soit (le clan, le quartier, le village, le groupe ethnique ou la nation) constitue un problème majeur de l'analyse (et de l'action) politique. Problématique qui mène directement à la question de la souveraineté, que Thomas Biersteker et Cynthia Weber proposent de penser comme un processus plus que comme un état de fait ${ }^{10}$. Autrement dit, ce concept englobe les actions constantes et répétitives que doit mener l'autorité pour démarquer l'espace physique, social et surtout symbolique sur lequel elle s'exerce, car son équilibre est toujours fragile, toujours menacé et ce, tant vis-à-vis de l'extérieur de la communauté, qu'à l'intérieur pour construire sa légitimité.

S'il faut donc produire continuellement cette communauté d'appartenance, une manière d'aborder la question des collections « extra- » ou « non-occidentales » pourrait consister à réfléchir aux usages qui en sont faits, précisément dans le but de rendre visible une collectivité dépositaire de cet héritage ou de cette culture. Cela revient alors à inverser la causalité la plus habituelle : au lieu de les considérer comme issus d'une culture, d'un groupe ethnique ou de toute autre entité préalablement constituée une fois pour toutes, il pourrait être productif de les analyser en tant qu'objets performatifs, capitaux, précisément, pour que le périmètre et les traits d'un groupe ou d'une communauté puissent commencer à apparaître, à être reconnus dans le champ politique. La question, dès lors, devient : quel sujet collectif prend forme derrière la catégorie "non-occidental ", et derrière ces collections ? Et, en contrepartie, comment se définit le sujet « occidental » face à qui la première catégorie s'érige ?

Pour offrir une analyse critique de ces positionnements identitaires - « nonoccidental » versus « occidental » - il est utile de les aborder en tant que « catégories géo-historiques », comme les nomme l'anthropologue Fernando Coronil ${ }^{11}$ : des représentations qui nourrissent notre sens commun et opèrent en reliant un lieu physique à une position fixe dans des rapports de pouvoir, et à une subjectivité (par exemple, I'idée de «tiers monde »). Plus important, ces représentations rendent opaque le sujet qui catégorise et la position à partir de laquelle il le fait. L'effet de vérité de ces classifications provient donc, au moins en partie, de leur caractère surplombant, du fait que le sujet occidental se présente comme le « point zéro » de l'observation. Par cette opération, "la différence se transforme en altérité12 », ce qui conduit Coronil à considérer de telles catégories comme des "fétiches de la modernité », dans la mesure où elles cachent les rapports qui les définissent. II en résulte que « des individus interconnectés en viennent à mener des vies séparées ${ }^{13}{ }^{\prime}$, les sujets étant compris et perçus comme appartenant à des unités closes et nettement différenciées. L'effort pour produire une analyse critique qui ne reproduise pas les valeurs et les hiérarchies véhiculées par ces " catégories géo-historiques » consiste à les détacher des significations ou contenus auxquels elles renvoient et à les situer comme les expressions non pas de "cultures », mais de rapports de pouvoir, et à observer ces rapports, tantôt conflictuels, tantôt consensuels, comme des interactions performatives, à travers lesquelles, justement, se façonne l'idée que deux espaces séparés existent.

Le cas mexicain est utile pour comprendre comment les polarités habituelles peuvent s'analyser comme l'effet de telles interactions, et non pas (ou pas seulement) comme leur cause. Dans ce cadre national - et malgré le sens commun dominant les frontières entre la nation et les populations qui s'identifient ou sont reconnues comme indiennes sont plutôt instables : parfois évidentes, parfois floues, on ne les trouve presque jamais là où on les attend. Depuis la fin de la colonisation espagnole en 1821, et encore plus depuis la Révolution (1910-1917), I'esprit républicain 
de la Constitution mexicaine ne reconnaît aucune fissure dans le corps citoyen, raison pour laquelle le périmètre de l'indigéneité ne s'établit pas sur une base légale. Ajoutons à cette première indétermination qu'au Mexique aucun « marqueur social d'identification » n'a jamais suffi à différencier de manière nette qui est Indien et qui ne l'est pas : ni la langue, ni l'habitat, ni les formes de subsistance, ni même le phénotype ne permettent de s'auto-identifier ou de reconnaître un autochtone sans équivoque (mais les identifications peuvent-elles jamais être univoques ?). Dans ce contexte, une analyse des objets associés à l'héritage précolombien ou à des expressions culturelles autochtones gagnerait, peut-être, à prendre au sérieux cette qualité indéterminée de l'altérité et à se demander si ces objets, en plus d'être des expressions culturelles, pourraient être vus comme des ancrages matériaux qui permettent de concrétiser cette identification, de fixer ces frontières, largement nébuleuses. En renonçant, en quelque sorte, à définir au préalable le groupe ayant produit ces objets, je propose plutôt de voir dans quelle mesure les rapports de pouvoir qui forgent en partie ces objets comme des formes d'expression culturelle produisent des dichotomies, des oppositions et des alliances permettant de rendre tangible une communauté d'appartenance, même si elle existe toujours dans la contingence et l'instabilité.

Vincent Négri. Sur le terrain du droit, la notion de collections extra ou non-occidentales ouvre sur un double registre où se croisent la question des qualifications juridiques et la temporalité. Du point de vue des catégories du droit et, partant, des qualifications, la notion ne fait pas sens : elle ne fait que renvoyer à d'autres notions, plus chargées juridiquement, comme celles de provenance et de territorialité - la provenance de la collection et la territorialité du droit applicable à la collection. En matière de revendication, la notion de collection extra-européenne est plus appropriée : les États membres de l'Union européenne sont liés, entre eux, par une règle commune en matière de restitution de biens culturels ; règle dont ne pourront se prévaloir les États extra-européens, le juge leur opposant alors la primauté de la loi nationale du territoire sur lequel se trouve le bien culturel au moment où la revendication est exprimée. De cette position du juge découle un rejet de la revendication exercée par un État extra-européen - État d'origine du bien culturel - lequel fonde son action sur sa législation relative aux biens culturels. D'où peut en être déduite une iniquité du droit.

Au surplus, la question du droit applicable doit être également appréciée au regard de la temporalité qui conduira à un traitement juridique différencié entre les frises du Parthénon, les objets du royaume de Bénin ou les butins militaires jusqu'à l'aube du XXe siècle, d'une part, et les pièces archéologiques issues de fouilles clandestines récentes, d'autre part. Les réponses du droit international sont articulées sur un principe de non-rétroactivité qui exclut du champ d'application des conventions internationales les faits commis antérieurement à l'entrée en vigueur de ces normes internationales. La codification du droit de la guerre, par les conventions de La Haye de 1899 et 1907, a formellement interdit le pillage (et donc la pratique du butin), de même que la saisie, la destruction, ou la dégradation des monuments et des œuvres d'art. En aval de cette immunité dont bénéficient, en droit, les biens culturels, c'est l'obligation de réparation en cas de violation de la règle qui présentera un revers positif ; la restitution de biens culturels pillés ou saisis lors d'un conflit étant une mesure de réparation. Les guerres coloniales sont incluses dans ce dispositif, mais ces normes ne peuvent être directement opposées à des situations antérieures à ces conventions. Ce faisant, cet ordre juridique, reflet du positivisme juridique et refuge de nombre de juristes, exprime - prolonge ? - une domination. Cette construction de la légalité internationale est excluante; elle constitue aussi un paravent, en occultant d'autres processus de création juridique. Car en contrepoint de la légalité, la légitimité ou l'équité peuvent orienter la formulation 
de solutions idoines, par le biais d'accords bilatéraux, voire guider l'interprétation jurisprudentielle, pour rendre compte des turbulences et des dissipations de la règle de droit. Ces voies-là ont été parfois empruntées. Sur le versant des restitutions de biens culturels, il s'agirait ainsi d'investir, aussi, l'impensé des juristes.

Viola König. Contrairement aux historiens, qui ont découvert ce sujet tardivement, les ethnologues et les chercheurs en science régionale se penchent sur ces collections muséales depuis des dizaines d'années. Ils évitent le terme de «non-occidental », qui implique une perspective euro-centrée et « appréhende des régions du monde dans leur seul rapport à l'Occident ». À l'inverse, I'histoire globale est une " histoire des interactions ». Elle se doit $d^{\prime}$ « argumenter de manière polycentrique et [de] prendre en compte des différences et des asynchronies régionales ${ }^{14} »$.

Les contextes d'acquisition respectifs sont tellement hétérogènes que chaque objet ou au moins chaque « collection homogène » issue du même contexte régional ou local nécessite obligatoirement une étude de cas détaillée et méticuleuse. La réduction à des catégories comme " butins militaires » ou " fouilles illégales » est incomplète et se révèle insuffisante dès lors qu'il est question de saisir des contextes individuels. Dans la majeure partie de ces contextes individuels, toute une série de revendeurs, de propriétaires successifs et d'intermédiaires locaux vient s'interposer.

Ariane Perrin. En effet, les Coréens par exemple ne se posent pas la « question de la restitution des collections non-occidentales » en ces termes-là. Ils souhaitent principalement que les biens culturels coréens qui ont été emportés illégalement puissent leur être rendus. Ils ne sont pas opposés à l'idée d'avoir des galeries d'art coréen dans des musées à l'étranger. Eux-mêmes financent l'ouverture et la promotion de ces galeries en y dépêchant parfois un conservateur coréen pour y travailler et valoriser ladite collection.

Je rejoins tout à fait Viola König lorsqu'elle parle de la nécessité d'étudier les modes d'acquisition au cas par cas car ils ont revêtu différentes formes et ont fait intervenir toutes sortes d'intermédiaires. Le manque de données précises sur les conditions de sortie d'un objet du pays d'origine est un écueil dans les demandes de restitution en Corée. On avance bien volontiers que ces objets ont été volés alors que le contexte précis de déplacement est le plus souvent inconnu. Ceci étant dit, pour la période coloniale en Corée (1910-1945), les autorités japonaises ont consigné dans des rapports officiels le problème endémique de pillage de sites archéologiques et de vols par tout un réseau de marchands d'art et d'antiquaires, ainsi que par des résidents locaux ${ }^{15}$.

Claire Bosc-Tiessé. La question de la restitution semble principalement posée dans le cadre des États-nations, entre anciens colonisés et colonisateurs, autour d'objets investis d'une charge symbolique qui cristallisent des enjeux identitaires et de construction nationale. Un mode d'articulation commun des notions de patrimoine, de mémoire, d'identité et de propriété ne s'est-il pas finalement établi, en miroir et dans la confrontation? Peut-on considérer qu'il serait partagé dans les grandes lignes par les parties prenantes, créant un terrain commun de négociation ou d'affrontement? Ces lignes peuvent-elles bouger et comment?

Paula López Caballero. La restitution d'objets s'inscrit effectivement dans un cadre éminemment national, et ce, à plusieurs niveaux. Premièrement, tel que mentionné dans les questions, ces objets sont investis d'une charge symbolique souvent exploitée dans les luttes de construction nationale. En second lieu, du moment qu'ils appartiennent à des collections publiques, ces objets font partie du patrimoine d'une 


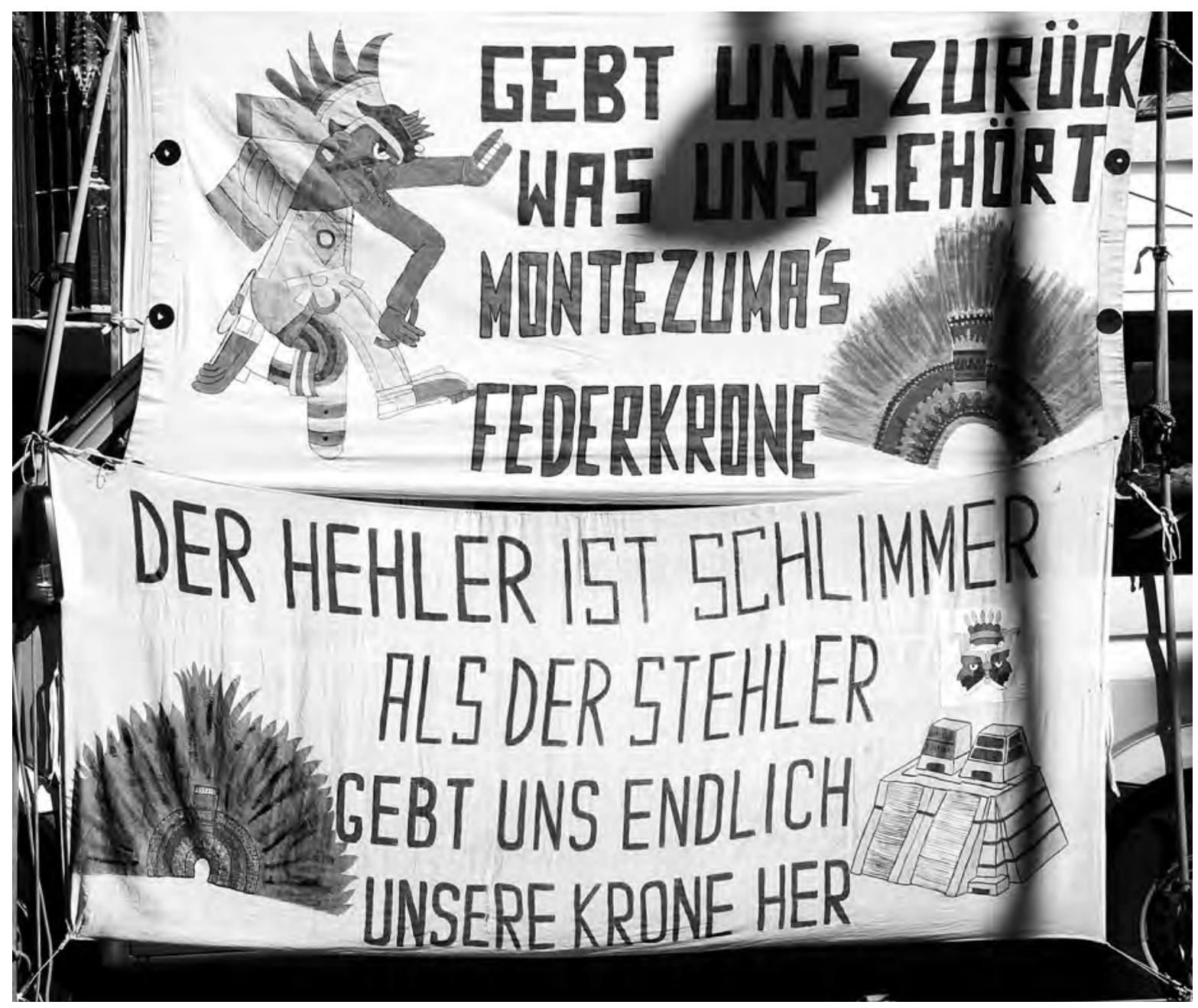

nation - souvent une ancienne métropole coloniale - et se trouvent généralement conservés et exposés dans des institutions publiques. Dans un tel cadre, ces pièces portent aussi une forte charge symbolique en tant que témoignages d'universalisme, d'ouverture au monde, voire de démocratisation, par exemple, autant de valeurs centrales dans la constitution du sujet « européen ». En troisième lieu, la restitution est un phénomène étroitement lié à l'origine même des nations : la plupart des demandes de restitution proviennent de pays issus des colonisations tardives, soit celles menées entre le XVIII ${ }^{e}$ et le XX $X^{\mathrm{e}}$ siècle, au moment où les États-nations européens eux-mêmes se consolidaient.

À ce sujet, encore une fois, l'Amérique latine, et plus spécifiquement le Mexique, offrent un contre-point de vue. Dans ces pays, il serait surprenant, voire hors de propos, de voir naître des actions en justice ou des mobilisations politiques contre l'ancienne métropole pour la restitution d'œuvres d'art ou de patrimoine archéologique. Lorsque des voix s'expriment contre un discours courant en Espagne qui présente l'évangélisation et la colonisation comme des actes de civilisation, la collectivité qui transparaît à travers celles-ci est bien la nation dans sa totalité, et non pas un groupe, " autochtone » par exemple, au sein de celle-ci. Peut-être plus surprenant encore, lorsque, par exemple,

1. Manifestation pour le retour de la coiffe de Moctezuma au Mexique, face à la Haas Haus, Stephansplatz, à Vienne, le 29 septembre 2007. 
des groupes de "néo-Indiens » dansent devant le Musée ethnologique de Vienne (Weltmuseum) pour exiger le retour au Mexique de la coiffe de plumes de Moctezuma, $c^{\prime}$ est bien au nom de toute la nation qu'ils le font (fig. 1). J'ajouterais que, de manière significative, ils ne proposent de remettre cette coiffe nulle part ailleurs qu'au Musée national d'anthropologie...

Mais alors, qui sont les acteurs des disputes à propos des collections « non occidentales » ou du patrimoine au Mexique? Il s'agit d'un champ de tension au sein même de l'État-nation, entre celui-ci et des populations identifiées comme autochtones ${ }^{16}$. Au Mexique, la mise en place progressive de ce que j'ai appelé ailleurs le « monopole légitime de l'État sur le passé et ses vestiges » a eu lieu pratiquement au même moment que les extractions mises en œuvre par les pays d'Europe dans leurs colonies ${ }^{17}$. Or, les coordonnées y étaient différentes : ces extractions (de la fin du XVIII au début du XX $X^{e}$ siècle) ont été menées par l'État sur son propre territoire, parfois contre l'avis des locaux. Elles ont aussi constitué une stratégie de défense de la souveraineté contre le pillage et l'exportation de pièces précolombiennes. À ce sujet, il est intéressant de rappeler l'intense débat, reconstitué par Haydeé López Hernández ${ }^{18}$, qui eut lieu à la fin du XIXe siècle pour établir l'origine des Mayas. Un certain nombre de chercheurs états-uniens ont alors défendu la thèse selon laquelle les Mayas avaient migré depuis le Mississippi et que, de ce fait, les ruines mayas en territoire mexicain étaient un héritage du voisin du Nord. La trajectoire historique menant vers un contrôle centralisé du patrimoine mexicain culmine, en quelque sorte, dans les années 1940, avec la création de I'Institut national d'anthropologie et d'histoire (INAH), chargé principalement de la protection, de la conservation, de l'étude et de la diffusion de tout le patrimoine national (principalement archéologique). Cet institut conjugue aussi l'aspect pratique des politiques publiques de protection et de diffusion du patrimoine avec la recherche scientifique. Et surtout, il a autorité sur tout le patrimoine, qui est donc compris comme un bien inaliénable de la nation. Cela, bien sûr, ne s'est pas déroulé sans conflits avec les localités où ces vestiges se trouvaient, faisant ainsi apparaître clairement la dimension politique et contentieuse des catégories d'identification (national, autochtone, occidental, non-occidental) : ce qui apparaît à l'échelle internationale comme un acte défensif devient, à l'échelle locale, un acte d'agression et d'imposition violente de l'autorité étatique, très souvent autour des mêmes objets.

Ariane Perrin. Les cicatrices de la période coloniale japonaise en Corée (1910-1945) demeurent trop profondes pour permettre d'entamer un dialogue apaisé sur la question des restitutions qui concernent également les objets qui auraient été « volés » à l'époque médiévale en Corée dès le XIV siècle. Le Japon est le pays qui possède le plus grand nombre de biens culturels coréens en dehors de la Corée, 74742 selon la Overseas Korean Cultural Heritage Foundation ${ }^{19}$. Qui plus est, sur les 160 peintures bouddhiques de l'époque Koryŏ (918-1392) - patrimoine rare et fragile qui a été redécouvert par les Coréens à partir des années 1970 et dont on pensait qu'elles étaient soit japonaises, soit chinoises - qui ont survécu, environ 130 sont conservées au Japon. La Corée possède moins d'une dizaine de ces peintures dans ses collections nationales. Du point de vue des Coréens, ce chiffre important pose la question des modes d'acquisition : ils invoquent les nombreux pillages et les raids de pirates - attestés par des sources historiques qui ont eu lieu en Corée au moment des invasions japonaises de la fin du XVI ${ }^{e}$ siècle ${ }^{20}$ mais aussi le transfert illicite d'objets d'art coréen pendant la période coloniale.

À l'heure actuelle, on observe plutôt une intensification des demandes de restitution de biens culturels coréens sortis illégalement du pays, qui est concomitante avec des demandes accrues d'inscription de sites patrimoniaux coréens sur la liste du patrimoine mondial de I'UNESCO. L'une des demandes de restitution concerne la question 
très sensible du retour en Corée de la collection de l'homme d'affaires japonais Ogura Takenosuke (1870-1964) qui possédait l'une des collections privées japonaises d'objets d'art coréens la plus importante pendant la période coloniale. Les autorités coréennes réclament son retour depuis 1945, arguant que les pièces archéologiques en particulier devaient provenir de fouilles illégales puisque des lois patrimoniales interdisaient leur exportation ${ }^{21}$. Comptant 1034 pièces dont plusieurs sont désormais considérées comme des biens culturels importants du Japon et 5 sont des " trésors nationaux », la collection Ogura a été léguée par ses descendants au Musée national de Tokyo en 1982. En juin 1965, un traité de normalisation des relations diplomatiques avait été signé par le Japon et la Corée du Sud qui a vu la signature de deux accords parallèles sur les compensations et sur le patrimoine. L'une des clauses de la partie coréenne abordait la question des biens culturels coréens « rapportés » au Japon pendant la période coloniale $^{22}$. Le Japon a restitué 1321 biens culturels coréens au moment de la signature de cet accord et s'en est depuis tenu là, estimant que l'accord bilatéral mettait un terme à toute demande de restitution d'objets déplacés pendant et avant la période coloniale. Jusqu'à présent les demandes de restitution couronnées de succès sont celles qui ont fait l'objet de négociations "privées " avec des institutions non gouvernementales et des collectionneurs. Dans ce cas, l'objet a été offert ou parfois acheté.

Le vol, ces dernières années, d'objets d'art coréens conservés dans des monastères au Japon est une conséquence inattendue de ces demandes de restitution infructueuses. Les personnes arrêtées d'origine coréenne ont indiqué lors de leur procès qu'elles ne faisaient que rendre à la Corée ce qui lui revenait, sans tenir compte d'autres contextes possibles d'exportation (cadeau diplomatique, commande, etc.) attestés par des sources historiques. Le dernier épisode de ce type concerne deux statues bouddhiques coréennes dérobées sur l'île japonaise de Tsushima en octobre 2012. Une statue a été rendue au Japon faute d'avoir pu identifier son propriétaire en Corée, tandis que la seconde, datant de l'époque Koryŏ, est réclamée par le monastère Pusŏk dans la province Sud du Ch'ungch'ŏng en Corée où elle aurait été consacrée en 1330. L'ordre bouddhique coréen du Chogye s'oppose à ce retour au Japon et la statue est, en 2018, toujours en Corée. Cet ordre est par ailleurs extrêmement actif dans les demandes de restitution. Après s'être rendu compte du vol de plusieurs centaines d'objets au cours d'un recensement des collections des monastères coréens à la fin des années 1990, l'ordre du Chogye a commencé à établir en 1999 une liste des objets disparus, "Stolen Buddhist Cultural Properties ", régulièrement mise à jour (couvrant la période 1984-2015) et dont une version anglaise est diffusée auprès d'organismes concernés à l'étranger. Deux musées américains ont ainsi rendu à la Corée, en 2013 et en 2017, deux peintures bouddhiques de l'époque Chosŏn (1392-1910). Depuis quelques années la presse coréenne se fait régulièrement l'écho de ces retours en Corée.

Laurella Rinçon. En ce qui concerne les biens culturels produits sur le continent africain ou dans les Amériques, sur lesquels je peux m'exprimer plus aisément, on peut considérer qu'ils symbolisent pour l'Europe une reconquête des pouvoirs spirituels perdus autant qu'ils sont, dans les pays d'origine, une composante majeure de l'identité des peuples dont ils incarnent l'histoire.

Dix-neuf des plus grands musées d'Europe et d'Amérique du Nord ont signé, en décembre 2002, la « Déclaration sur l'importance et la valeur des musées universels ${ }^{23}$ ", en prévention d'un débat sur les restitutions appelé à s'intensifier. Ranimant de ce fait une polémique déjà ancienne, mais encore vivace, ils exprimaient une volonté d'aborder la question selon une communauté de vision où patrimoine, mémoire, identité et propriété seraient dans un cas de l'ordre du partage entre tous et dans l'autre de l'ordre de l'appropriation entre soi. Il est intéressant de noter la réponse du philosophe 

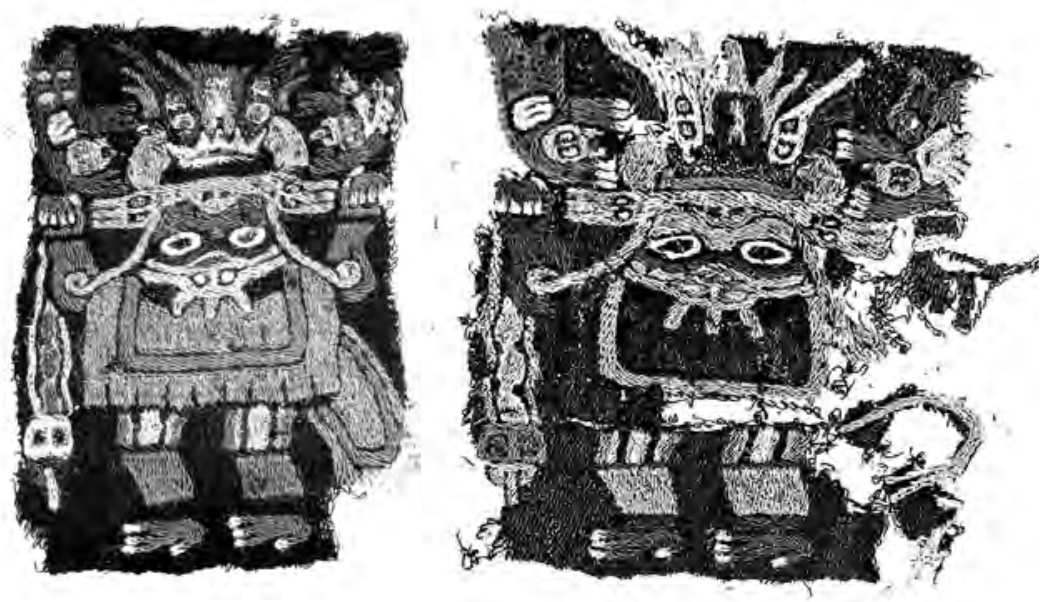

Édouard Glissant à cette posture du musée universel : "L'universel qui est une grande conception de l'Occident, une sublimation même, ne correspond pas à notre réalité. L'universel est la sublimation du particulier $^{24}$. » Elle fait écho à celle du professeur George Abungu, archéologue et directeur général émérite des Musées nationaux du Kenya, qui note que ces derniers possèdent la plus grande collection d'hominidés au monde et n'ont pas été invités à faire partie des musées universels. II s'interroge donc sur le fondement de I'universalité des musées signataires,

2a-b. Motifs brodés provenant d'un tissu détruit, faisant partie d'une série de 24 fragments (inv. 1935.32.0213), Pérou (Paracas), Göteborg, Världskultur Museerna (Musée des cultures du Monde). tous situés en Europe : "Tous les musées ne partagent-ils pas une vocation et une vision communes $^{25} »$ et, de ce fait universelle?

Il semble toutefois important de noter qu'à ce jour, il y a en réalité plus de cas de restitutions résolus, suite à l'expression d'une demande, que de cas de crispations. Depuis vingt ans, les expériences ont été nombreuses, et des institutions ont nourri le débat en ouvrant le dialogue avec les communautés réclamant le retour de leurs biens culturels. En Suède, par exemple, le Musée des cultures du monde de Göteborg a développé des projets de collaboration avec les institutions péruviennes, aboutissant à la restitution des textiles Paracas (fig. 2a-b).

Par ailleurs, les grandes expositions rétrospectives contribuent à susciter un désir de retour des objets dans leur lieu d'origine, autant au niveau des États que des populations qui ressentent un sentiment de trop grand éloignement du patrimoine incarnant leur culture. Ce fut le cas à l'occasion de l'exposition Paracas: A Stolen World ${ }^{26}$ qui a déclenché en 2008 I'intérêt des autorités du Pérou. Le processus de restitution a ainsi débuté peu de temps après, en 2013, et s'est conclu par un accord entre les gouvernements péruvien et suédois, en 2014, pour le retour d'un ensemble de 89 textiles Paracas. De même, l'exposition Les forêts natales, arts d'Afrique équatoriale atlantique ${ }^{27}$, ouverte au musée du quai Branly en 2017, et qui proposait de révéler la créativité et I'originalité des arts de cette région à travers une sélection d'œuvres emblématiques, a suscité quelques mois plus tard des revendications du Gabon. Le ministre de la Culture du Gabon a annoncé la création, le 8 juin 2018, d'une Commission nationale chargée du rapatriement des œuvres gabonaises, travaux auxquels est associé l'un des commissaires de l'exposition, I'historien de l'art Jean-Louis Paudrat.

Vincent Négri. Ne retenons dans l'énumération des notions que deux d'entre elles, le patrimoine et la propriété - notions cardinales dans le contexte que vous évoquez qui ont un contenu juridique fort. Elles nous sont tant familières que nous les pensons universelles, et susceptibles de délimiter un terrain commun. Mais ces deux notions sont d'abord les matrices d'une culture juridique occidentale, qui a certes essaimé, mais dont l'universalité est loin d'être acquise. Des travaux de recherche récents ont mis en évidence que la notion de patrimoine, de même que les catégories et les qualités qui la forgent (dont la notion de propriété), ne sont pas identiques d'une culture et d'une langue à l'autre - la langue restant elle-même le bien patrimonial le plus profondément lié à l'identité des peuples - et que si le patrimoine se décline en objets, en monuments, en richesses animale et végétale, en traditions et récits, il se dit surtout 
en « mots » : ce sont des mots dans les langues qui ancrent les patrimoines dans les cultures $^{28}$. Or, pour affecter un régime juridique à un bien, le droit procède en deux temps, d'abord par la désignation de l'objet, puis par l'énoncé de la règle de droit. Le droit nomme, puis norme l'objet nommé. On perçoit ainsi la difficulté à établir un dialogue en imposant un vocabulaire et un ordre juridique dont aucune traduction, ni équivalent, n'existe dans la langue et la culture de certaines communautés. Pour autant, si les passages d'une langue à une autre supposent des pertes, ils autorisent aussi des innovations; à chaque fois que s'engage le processus de traduction, faire le bilan de ce qui résiste, de ce que l'on perd, de ce que l'on gagne, permet de s'approcher au plus près des singularités culturelles. À titre d'exemple, les acceptions du mot patrimoine dans les langues africaines dérivent d'une langue à l'autre et la notion de conservation du patrimoine - le paradigme de la conservation est matriciel dans notre conception occidentale du patrimoine culturel - peut varier dans certains contextes socio-culturels : ce ne sera pas tant l'intérêt reconnu au bien, mais les conditions d'acquisition ou de transmission qui prévaudront. Il pourra en être ainsi de la chose acquise sans souffrance, qui ne sera pas conservée, car si la facilité en commande l'acquisition, il n'y a pas lieu d'en prendre soin. La connaissance de ces différenciations linguistiques peut aussi contribuer à identifier les canaux d'échange et de partage permettant d'éviter les chocs entre patrimoine des uns et patrimoine des autres.

Sur la question des restitutions, c'est aussi la notion d'État-nation qui est mise en tension. Le temps où le contrat social international reposait sur la seule souveraineté des États-nations semble révolu ; un mouvement en faveur d'un nouvel équilibre des rapports composant ce contrat social s'exprime et l'évolution contemporaine du droit international du patrimoine culturel en est un symptôme. Ce déplacement du centre de gravité du droit international, dans une galaxie où la société civile conteste aux États la légitimité de les représenter, dessine une autre vision des revendications de biens culturels par des communautés. C'est en même temps un droit à la différence qu'expriment les demandes de restitution de biens culturels portées par des communautés infra-étatiques ou transnationales.

Benoît de L'Estoile. La notion de " patrimoine national », souvent associée à l'inaliénabilité, renvoie au double registre juridique de la propriété et de la souveraineté. Dans la tradition occidentale (qui domine aujourd'hui les relations internationales), ces deux modes apparaissent comme caractérisés par l'exclusivité : la propriété est « le droit de jouir et disposer des choses de la manière la plus absolue »; la souveraineté sur un territoire est difficilement conçue sur le mode du partage. Dans une telle logique binaire, il n'y a pas d'alternative autre que la conservation (légitime) d'un objet, ou sa restitution. La demande de possession exclusive possède aussi une dimension politique. Les demandes de restitution sont ainsi souvent associées à des revendications identitaires, qu'elles soient nationalistes, régionalistes, religieuses ou ethniques. Pourtant, les cas de restitution montrent les difficultés et les tensions entre différents acteurs sur les lieux « d'origine », en particulier du fait des incertitudes autour de la définition des « héritiers » ou « ayants-droit » (''État-nation, le groupe ethnique, le clan, les descendants, souvent multiples, parfois divisés, etc.).

Emmanuel Kasarhérou, aujourd'hui directeur-adjoint des collections au musée du quai Branly, racontait que, alors directeur du musée de Nouvelle-Calédonie, à Nouméa en 1990, il avait organisé une exposition d'objets kanak conservés dans des musées français et européens, De jade et de nacre ${ }^{29}$. Il avait consulté un «vieux ", de son propre clan, dans la tribu de Houaïlou, à propos d'une "tête de monnaie » (objet rituel kanak) qui avait été donnée au début du XXe siècle au missionnaire protestant Maurice Leenhardt. Le vieux répondit au directeur du musée, inquiet devant la possibilité 
de faire face à la demande que l'objet soit retourné au clan : "Je suis content de voir cet objet, mais il a probablement été donné dans des conditions particulières. Ce qui a été noué à un moment, donné par la parole, ne peut pas être dénoué si l'on n'en connaît pas le vocabulaire et la syntaxe. »

L'image du « nouer » invite à penser un objet de musée en tant qu'il est un nœud de relations qui se tissent à travers I'histoire. L'idée de « lien » me semble intéressante de par sa polysémie : un lien, c'est ce qui attache, ce qui emprisonne ; c'est aussi ce qui rattache, ce qui relie. Il est peut-être possible de transposer cette image en parlant de "liens coloniaux ». Les "liens coloniaux » renvoient à ce qui a été noué dans le passé, mais aussi (en tout cas c'est une possibilité) à ce qui continue de relier.

Si le retour des objets dans leur pays d'origine ("la restitution») constitue la forme en apparence la plus évidente - sinon la plus simple à mettre en place en raison des difficultés théoriques et pratiques qu'elle suscite - il faut dépasser l'idée que c'est là la seule forme de réappropriation possible. Le défi est d'imaginer de nouvelles voies de réappropriations. Parler en termes $d^{\prime}$ « héritage colonial » implique qu'il y a un héritage partagé, ce qui ne veut pas dire consensuel ; le conflit est aussi un mode de partage. C'est ce que suggère le tableau du peintre congolais Chéri Samba (fig. 3), qui imagine que la statue de I'Anyota (homme-léopard), pièce mythique du Musée royal de l'Afrique Centrale de Tervuren, près de Bruxelles, est l'objet d'un conflit entre ceux qui veulent le faire sortir du musée et ceux qui veulent qu'il y reste. Le texte en lingala sur le tableau précise que ceux qui sont en dehors sont « un groupe d'Africains décidés à ne plus voir ce genre de sculpture déshonorant l'homme noir », tandis que le groupe des employés (blancs) du musée déclare : « Nous ne pouvons accepter que cette œuvre parte, c'est elle qui a fait ce que nous sommes aujourd'hui. »Cette caractérisation invite les divers héritiers non à s'ignorer, mais à s'efforcer de trouver un compromis, un modus vivendi.

Viola König. Les ethnologues et les spécialistes de science régionale travaillant dans des musées cherchent en premier lieu à établir un contact avec les descendants des sociétés desquelles proviennent ces objets - ou bien ce sont eux qui sont contactés par les familles. Une médiation, qui se prolonge parfois durant plusieurs années, permet de négocier les meilleures conditions de maintien des objets dans les collections muséales, ainsi que celles de leur conservation, par exemple la question de leur restauration, les tabous qu'implique leur présentation lors d'expositions, etc., autrement dit tous les aspects susceptibles de restreindre leur accès, qui sont définis selon des critères culturels et tributaires du Zeitgeist mais qui sont susceptibles de changer en permanence.

À mes yeux, la fonction des musées ethnologiques consiste à aider les mandataires des sociétés d'où proviennent les objets, à formuler des demandes de restitution de manière professionnelle afin d'éviter qu'elles soient rejetées pour des raisons de forme ou de contenu. L'objectif est de prendre une décision juste. Afin de s'assurer que des restitutions ne bénéficient pas à des destinataires illégitimes, les personnes réclamant la restitution des objets ont l'obligation de prouver la légitimité de leur revendication si celle-ci ne ressort pas d'emblée clairement des sources écrites dont on dispose. Cette légitimation est nécessaire car dans certains cas, lorsque des représentants de pays occidentaux ont pris en charge la requête formelle, il est important de s'assurer qu'ils détiennent bien l'autorisation des sociétés concernées pour le faire.

Claire Bosc-Tiessé. Dans ce contexte, est-ce que les dépôts ou les prêts à long terme peuvent par ailleurs être satisfaisants symboliquement ou comment répondraient-ils, de ce point de vue, à la demande de restitution? 


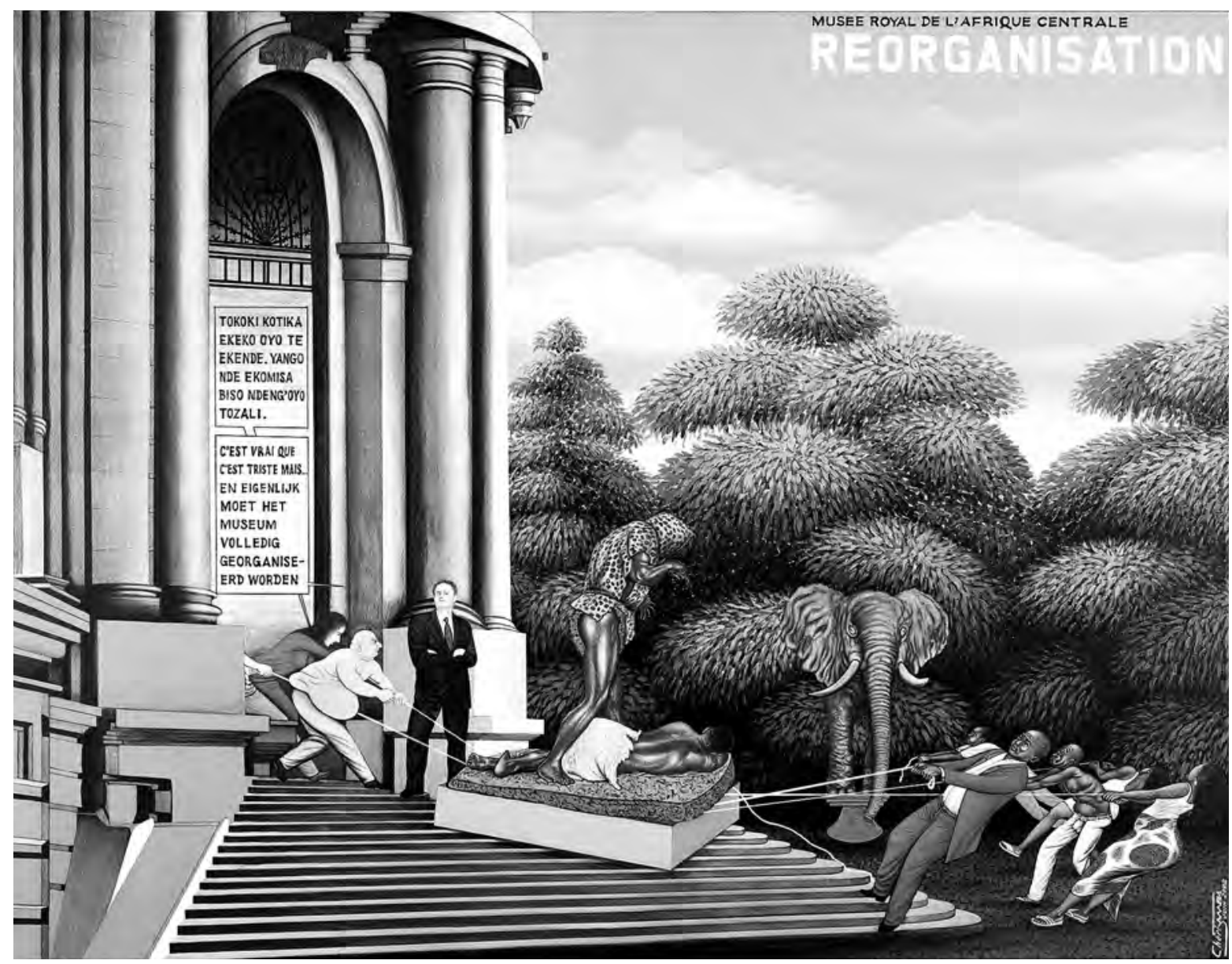

Viola König. Tout dépend de ce que vous entendez par « dépôts ». Si des collections restituées ne sont, par exemple, qu'entreposées dans un musée national australien et qu'elles ne sont pas directement redonnées aux aborigènes afin qu'ils puissent en avoir I'usage, les restitutions sont peu pertinentes. Dans ce cas, elles ne servent en réalité que les intérêts de l'État qui détient les collections et aspire à une politique de réparation.

Nous gardons en permanence à l'esprit la cause des descendants des sociétés $\mathrm{d}^{\prime}$ où proviennent ces objets. Il est important que les revendications de restitution trouvent une issue qui soit favorable aux mandataires de ces sociétés ou des communautés qu'ils représentent. Étant donné que la conservation de matières organiques peut être onéreuse, on pourrait par exemple négocier des contrats de prêts permanents. Dans ce cas, il faudrait procéder à une modification du statut d'ayant-droit/propriétaire : les musées européens prendraient en charge fiduciairement la conservation des collections pour le compte des sociétés d'où proviennent les objets en question, mais n'en seraient plus les propriétaires et auraient l'obligation de les restituer à tout moment sur simple demande des sociétés concernées.

Benoît de L'Estoile. Dans le cadre des réflexions actuelles sur le retour des objets ethnographiques vers leur pays d'origine, les musées ne sont plus vus comme les détenteurs, au nom de la nation, possesseurs uniques de ces collections inaliénables. Souvent,
3. Chéri Samba, Réorganisation, 2002, Tervuren, Musée royal de l'Afrique centrale. 


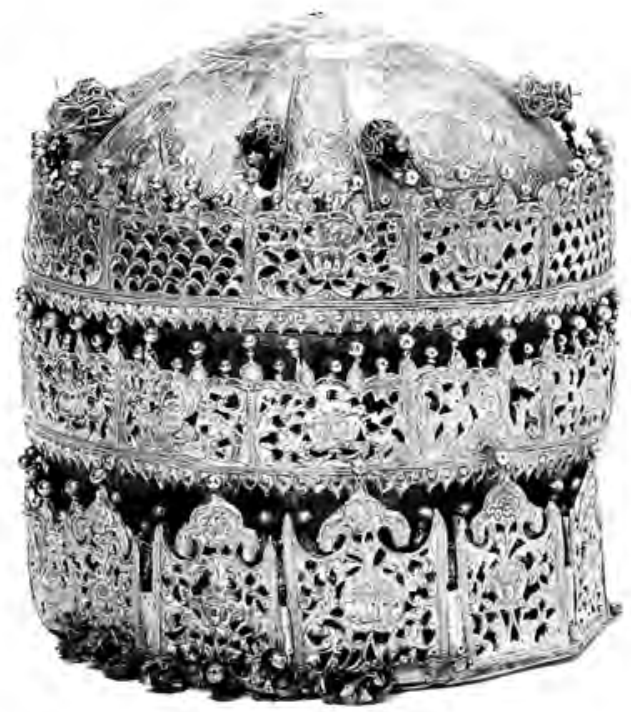

ils sont plutôt perçus comme des gardiens (stewards en anglais), comme des conservateurs au profit des communautés sources. Du moins la question du partage du patrimoine se voit-elle posée.

Marie-Claude Tjibaou, veuve du leader indépendantiste kanak Jean-Marie Tjibaou, contestait en 2007 I'idée selon laquelle « ce patrimoine dispersé devrait faire I'objet d'un rapatriement systématique en NouvelleCalédonie ». Elle suggérait plutôt que «la présence effective de ces objets kanak dans les grands musées nationaux, européens ou américains, constitue aujourd'hui une nouvelle forme de représentation de notre culture. Ces objets deviennent en quelque sorte nos ambassadeurs de par le monde, ils portent avec eux notre parole d'hier et celle d'aujourd'hui. »Cette proposition ouvre une piste intéressante, en ce qu'elle articule une alternative ou un complément à la " restitution », en transformant le sens de la présence dans les musées « occidentaux » d'objets originaires d'un territoire ou d'une culture, en établissant la revendication d'une forme de "droit de regard », et d'un

4a. Couronne pillée dans le camp royal de Maqdala, vers 1740 ?, Éthiopie, alliage d'or, d'argent et de cuivre filigrané, perles de verre, cuivre coloré et doré, Londres, The Victoria and Albert Museum, M.27-2005. lien d'appropriation. Si les objets sont des " ambassadeurs », ils doivent par là-même des comptes à leurs « mandants »; ils deviennent porteurs d'un « message » de ceux-ci aux institutions muséales du « Nord » et aux visiteurs de celles-ci. De fait, fut alors mis en place un programme au Centre culturel Jean-Marie-Tjibaou de Nouméa, intitulé "Objets ambassadeurs », afin de permettre la venue provisoire (pour un ou deux ans) d'objets venus des grands musées européens ${ }^{30}$.

Seule une très faible partie des 300000 objets conservés dans les collections du musée du quai Branly est aujourd'hui montrée sur le plateau des collections (3 500 environ). Ceux qui sortent des réserves pour des expositions temporaires, sur place ou dans d'autres musées, sont aussi en effectif réduit. Un nombre considérable d'objets restent donc « disponibles » pour donner lieu à des prêts à moyen ou long terme dans des musées non européens. Certes, il existe des contraintes matérielles à la circulation des objets, notamment en termes d'accueil, mais il convient de chercher des solutions pratiques. Surtout, ces arrangements ne doivent pas être envisagés sur un mode unilatéral : on peut imaginer de reconnaître au pays d'origine la propriété éminente d'un objet aujourd'hui dans des collections européennes, et négocier son prêt à long terme pour qu'il soit exposé, sur le modèle des têtes nok en dépôt par le Nigéria au musée du Louvre.

Certains musées évoquent la « restitution virtuelle» comme une panacée. Le terme est malencontreux, dans la mesure où l'on ne peut manquer de suspecter qu'il s'agit de « rendre » un ersatz, une « reproduction », tout en conservant la substance. Cependant, dans le domaine des images par exemple (photographies, dessins, films, etc.) ou des sons (musique, parole, etc.), la numérisation et la diffusion virtuelles peuvent constituer des vecteurs de réappropriation efficaces, dans la mesure où la question de l'original n'est pas constituée en enjeu essentiel.

Laurella Rinçon. Le Victoria and Albert Museum a proposé d'initier, à l'occasion de I'exposition Maqdala 1868, ouverte en 2018, des prêts à long terme avec les institutions patrimoniales éthiopiennes. Cette exposition raconte, à travers vingt objets, I'histoire d'une expédition britannique qui eut lieu sous le règne de l'empereur Tewodros II et entend s'interroger sur l'histoire de ces objets en retraçant leurs origines. Les autorités éthiopiennes ont accueilli cette proposition en formulant en réponse une demande de 
retour sans conditions du butin de Maqdala, composé de pièces remarquables, fruits d'une histoire complexe, emportées par l'armée britannique pendant l'expédition d'Abyssinie en 1868, et conservées au Victoria and Albert Museum depuis 146 ans (fig. 4a-b). Les prêts et dépôts à long terme relèvent donc de la question de la circulation des biens culturels, qui est une problématique différente de celle de la propriété.

Lorsque le président de la République du Bénin, Patrice Talon, souligne le $1^{\text {er }}$ juin 2018, dans son discours d'ouverture de la conférence internationale de I'UNESCO sur la circulation des biens culturels ${ }^{31}$, que « ces biens uniques et exceptionnels, dont I'histoire a du mal à être contée avec aisance dans leurs lieux actuels de rétention, n'attendent que d'être retournés dans leurs environnements naturels en vue de leur renaissance », il engage fermement la discussion sur la question de la propriété. Bénédicte Savoy, dans son allocution lors de la même conférence ${ }^{32}$, oppose également les questions de partage du patrimoine et celles de propriété, posant clairement, dès l'introduction, les termes du débat. II semble difficile, face à l'expression d'une demande si précise, de proposer en réponse des dépôts et des prêts à long terme. Le président Patrice Talon ajoute que « ces biens ont une âme et que revenus sur leur terre de création, exposés parmi les leurs, là où tout est en cohérence avec leur essence et où leur histoire révèle davantage leur grandeur que leur asservissement, ces témoins exceptionnels de notre histoire vivront enfin le rayonnement que vivent leurs semblables des autres contrées de ce même monde [...]. De ce fait, ils doivent rester la propriété de ces peuples tout en continuant d'être accessibles à toute personne en quête de découverte et de savoir. » Nombreuses sont les voix qui convergent donc pour réclamer une discussion sincère et équitable sur la question de la propriété avant que ne soient envisagés des projets de collaboration qui pourraient favoriser la mobilité des biens culturels.

Vincent Négri. Le dépôt ou le prêt à long terme sont des simulacres de restitution, ou un art de transposer les référents juridiques de celui qui prête dans l'univers culturel de celui qui, en apparence, bénéficie d'un retour de l'objet ; ou encore, et autrement dit, un art d'imposer son système juridique et, partant, ses valeurs, à autrui. Ce faisant, on raisonne en termes de perte et de profit, et le droit, notamment le droit du patrimoine, favorise ce mode de pensée. Il n'est pas déraisonnable d'envisager de construire un autre rapport juridique, où les deux parties sont du côté du gain. Mais cela induit de faire prévaloir l'accès du public et des communautés, la connaissance partagée, la coopération et la collaboration scientifiques, sur des questions de propriété et d'exclusivité. Des modèles existent ${ }^{33}$, mais $c^{\prime}$ est peu dire qu'ils ne sont pas partagés...

Ariane Perrin. Dans le cas de la Corée, la demande de restitution des 297 protocoles royaux illustrés de la période Chosŏn, les ŭigwe ou « Livre des rites d'État », actuellement conservés par la Bibliothèque nationale de France, et qui font l'objet d'un contentieux entre la Corée et la France depuis leur redécouverte en 1975, est exemplaire. Ces archives 4b. Robe pillée dans le camp royal de Maqdala, années 1860 ? Éthiopie, coton brodé de soie, Londres, The Victoria and Albert Museum, 399-1869. 


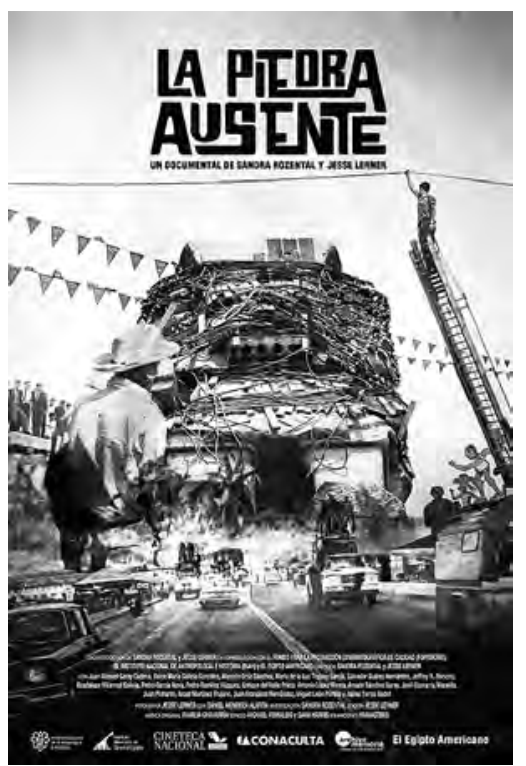

5. Affiche du film documentaire de Sandra Rozental et Jesse Lerner La Piedra Ausente, INAH / IMCINE, 2013. royales ont été saisies à la bibliothèque royale sur l'île de Kanghwa en 1866, lors de l'expédition punitive des troupes françaises en représailles de l'exécution de missionnaires catholiques français la même année. Ces documents historiques revêtent une importance particulière en Corée car non seulement il s'agit de copies (supérieures en qualité d'exécution) qui étaient réservées au roi mais, surtout, une trentaine de ces livres sont des exemplaires uniques. Selon les termes de l'accord relatif aux manuscrits royaux de Chosŏn qui a régi le prêt de 2011 entre la France et la Corée, ces manuscrits « font partie de l'identité du peuple coréen et constituent un élément fondamental de la mémoire de la Corée du Sud ${ }^{34}{ }$. Cet accord prévoit le retour des documents en Corée sous la forme d'un prêt de cinq ans, renouvelable. Ce rapatriement a été fortement médiatisé en Corée ; plusieurs expositions ont eu lieu, accompagnées de la publication de catalogues ${ }^{35}$. La formule du retour partiel n'est pas satisfaisante pour la partie coréenne qui souhaite une restitution définitive, d'autant plus que celle-ci avait été au cœur d'une transaction commerciale en 1993 quand les Coréens avaient accepté d'accorder à la France le marché du TGV en Corée en échange de la restitution de ces archives royales.

Paula López Caballero. Le problème de la circulation des collections «non occidentales » ou de la constitution contemporaine de ce type de collections se pose avec encore plus d'acuité, sans doute, lorsqu'il s'agit de restitutions entre deux pays. Dans un même contexte national, comme celui du Mexique, signalons quelques stratégies qui ont été mises en place par les institutions muséales. Manuel Burón Díaz mentionne que la crise politique de 1968 touchait déjà la pratique archéologique et les structures institutionnelles de conservation du patrimoine ${ }^{36}$. L'un des principaux questionnements était alors la spoliation que l'État fédéral, à travers I'INAH, semblait exercer envers les localités pour y extraire des pièces archéologiques, ou exproprier des terrains lorsqu'un site archéologique y était découvert. Inspirée par les écomusées français, la conférence de I'UNESCO tenue à Santiago du Chili en 1972 établit comme principe directeur la création de musées au service de la communauté. La même année, au Mexique, la Loi fédérale sur les monuments et les zones archéologiques, artistiques et historiques, fut réformée, ouvrant ainsi la possibilité, pour les gouvernements locaux, de sauvegarder le patrimoine, sous certaines conditions : ainsi naissent les musées communautaires, particulièrement dans l'État d'Oaxaca. Toujours selon l'historien, ces musées ne constituent pas une rupture radicale avec le passé, car depuis la création de l'INAH dans les années 1940, les archéologues ont toujours eu recours, de façon informelle, au soutien local. Ils offrent cependant la possibilité de conserver et d'exhiber des pièces archéologiques dans la région même où elles ont été trouvées, limitant ainsi le centralisme qui caractérisait l'institution jusque-là.

Pour sa part, I'anthropologue et cinéaste Sandra Rozental documente, d'un point de vue ethnographique, les rapports de pouvoir et les effets provoqués par le retrait du plus grand monolithe précolombien du Mexique - le Tláloc - du village où il se trouvait, dans les années $1960^{37}$ (fig. 5). La contestation locale ne s'est pas fait attendre. Entre le mythe autour des pouvoirs associés à Tláloc - le dieu de la pluie selon la cosmogonie aztèque -, des conflits entre factions au sein du village, un important investissement technique pour le déplacer et des confrontations ouvertes avec l'armée, cet épisode témoigne de la récupération « par le haut » des arguments conservateur et nationaliste. Si le cas du Tláloc est exceptionnel non seulement de par l'importance des ressources que l'État y investit, mais également par la durée de la réaction locale, le type de rapport qui s'y établit entre localité et État est, selon toute probabilité, assez courant. La recherche de Rozental est aussi intéressante d'un autre point de vue : à l'issue de ce conflit, 
I'INAH s'est engagé à remettre une réplique du monolithe à la municipalité, attribution actée, finalement, en 2007, ce qui ouvre tout un ensemble de questionnements sur le vrai et le faux, I'authentique et la copie, et sur la perception différentielle de la valeur attribuée à cette pièce. En effet, il s'agit d'une copie " conforme » ou officielle de la statue, fabriquée par l'institution même chargée de différentier l'authentique du faux. En même temps, il est courant de parler de cette statue comme du « retour du Tláloc au village », effaçant ainsi cette limite structurante entre le vrai et le faux et remettant en cause, en quelque sorte, la valeur primordiale donnée à l'ancienneté de la pièce originale pour privilégier, enfin, la fierté locale.

Je donnerai un troisième exemple, qui procède, en revanche, $d^{\prime}$ 'une initiative individuelle : il s'agit d'une œuvre d'art d'un artiste contemporain mexicain, Eduardo Abaroa, intitulée Destruction totale du Musée d'anthropologie ${ }^{38}$ : un lieu hautement significatif, ce musée est conçu comme une synecdoque, autant de la nation ellemême que de l'appropriation symbolique - et matérielle - par l'État et par la nation de I'héritage autochtone, précolombien et contemporain. L'œuvre est composée autant de pièces abstraites, que de plans avec des instructions précises pour dynamiter le Musée national d'anthropologie et surtout d'un montage suggestif de photographies prises pendant la construction du musée et conservées dans ses archives. L'artiste en a fait des tirages de grand format qu'il a entourés de restes de briques, de ciment et de tiges d'acier - invitant le public à imaginer la démolition violente du musée précisément avec les images de sa construction (fig. 6).

Si les deux premières situations - musées communautaires et copies officielles ne supposent aucune remise en question de l'autorité muséale et des principes qu'elle diffuse mais, simplement, de mieux distribuer les ressources, en revanche pour l'artiste
6. Eduardo Abaroa, ve de l'installation Destrucción total del Museo de Antropología, Mexico, kurimanzutto, 2012.

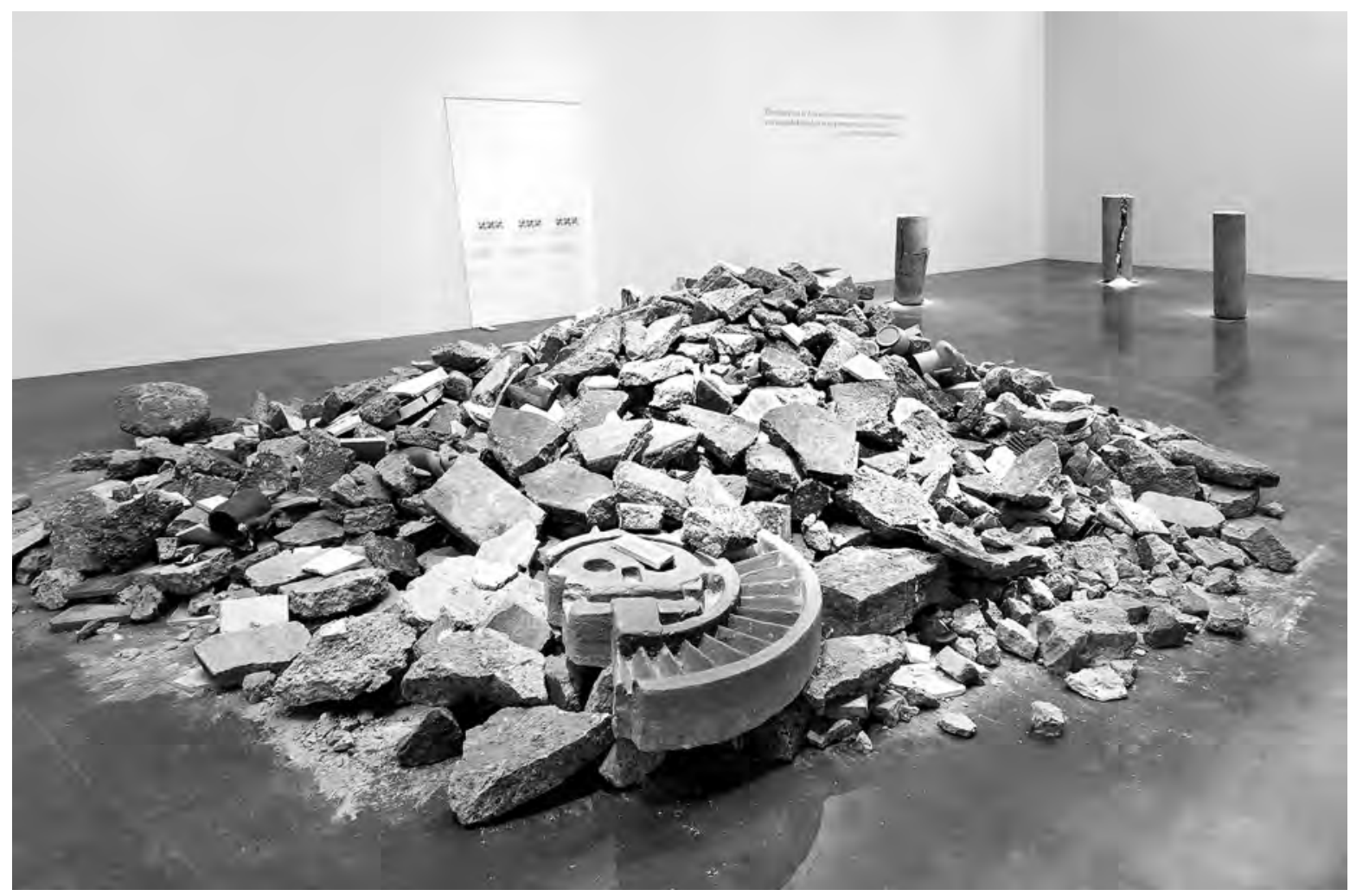


il n’y a pas de solution intermédiaire. À travers ses pièces, Eduardo Abaroa sublime sa proposition comme unique solution : une destruction totale du musée afin de permettre sa refondation sur de nouvelles bases. Cette intervention artistique me semble plus que nécessaire pour démonter l'effet de vérité produit par le musée. Pour être inclusif, toutefois, le patrimoine n'est pas obligé de disparaître. De manière générale, tout élan productif devrait aller dans le sens d'une dilution de la « voix d'autorité » de l'institution muséale, incluant le rapport privilégié et presque sacré que, souvent, les conservateurs entretiennent avec les objets, en resituant continuellement leurs points de vue dans des rapports de pouvoir et dans le temps.

Plus spécifiquement, ce qu'une perspective d'anthropologie politique peut offrir pour penser de manière critique le devenir de ces collections "non-occidentales» porte sur les bases conceptuelles, les prémisses sous-tendant l'idée de « collection non-occidentale ». En réaction à l'effet de naturalisation que produisent les musées, un premier principe consiste à expliciter ouvertement les échanges, les conflits et les interactions ayant conditionné la constitution même de la collection. Qu'est-ce qui a permis d'acquérir un certain nombre d'objets ? Le musée étant une institution disciplinaire, dans le sens que Michel Foucault a donné à ce terme, c'est-à-dire, qui contribue fortement à renforcer des normes sociales qui façonnent et délimitent les subjectivités, un second principe consiste à réfléchir à des mécanismes permettant de rendre visible non seulement l'historicité des collections, mais également la contingence des significations attribuées par le musée à ces pièces. Comment les collections contribuent-elles à créer une identité, un passé et un héritage, même en porte-à-faux lorsqu'il s'agit, par exemple, de pièces issues des expéditions coloniales? Qu'est-ce qui permet de classifier les pièces dans un certain ordre, de privilégier certains critères, d'en exclure d'autres? Quelles conditions matérielles et intellectuelles permettent de transformer un objet d'usage sacré ou quotidien en œuvre d'art ? II s'agit de donner à voir, en somme, les « échafaudages » qui soutiennent la scène principale, et d'inviter ainsi à prendre conscience de la contingence et de l'historicité des discours mobilisés à chaque exposition. Une conséquence de cet exercice d'explicitation serait, également, d'ouvrir le musée à la possibilité de multiplier les significations des mêmes pièces, notamment lorsque celles-ci n'ont pas été conçues comme des œuvres d'art ou des pièces destinées à être exposées.

Claire Bosc-Tiessé. C'est un sujet où le chercheur en sciences humaines et sociales est directement confronté à des controverses et à des contentieux aux implications politiques, morales, idéologiques et économiques. Comment articule-t-on la démarche scientifique et l'intervention publique? La recherche parvient-elle à se faire entendre dans le débat public?

Benoît de L'Estoile. Souvent, le chercheur s'efforce de donner le sens de la complexité, alors que le débat public (notamment dans sa version télévisée) favorise au contraire les positions tranchées et simplistes. Du point de vue des chercheurs, I'invitation sur les plateaux de télévision de publicistes dont le raisonnement ressemble à des slogans est un peu décourageante. La question de la "violence coloniale », par exemple, qui constitue un objet de recherche et de réflexion important, peut conduire à occulter la multiplicité des formes d'interaction et d'appropriation au sein du rapport colonial. Paradoxalement, cette insistance sur la violence fait des colonisateurs les seuls acteurs, les colonisés étant condamnés à la seule alternative entre "résistance » et " soumission ». Une telle alternative revient à leur dénier toute capacité de réflexion et d'action autonome, c'est-à-dire à les réduire à leur seule condition de « colonisés ». 
Cependant, les questions des chercheurs, présentées dans des ouvrages, des séminaires, des conférences, ont un impact dans le milieu des musées, et contribuent à transformer la muséographie, comme on le voit au musée du quai Branly, qui a transformé la section Amériques pour tenir compte de certaines critiques, ou dans d'autres cas. Il y a donc un vrai enjeu à ce que les chercheurs fassent l'effort de s'adresser à un public large.

Laurella Rinçon. De nombreux cas résolus démontrent que c'est bien la recherche sur les collections, et la connaissance et le savoir développés sur les objets qui ont permis de dépasser la controverse et d'aboutir à une solution viable pour les deux parties. La recherche est d'ailleurs l'un des éléments mis en avant dans les protocoles de résolution de cas de restitutions, où les deux parties prolongent leur discussion en développant des projets de partenariats et, notamment, des programmes de recherche communs sur les collections. Ces derniers s'accompagnent souvent de plans de formation et de renforcement de capacités des personnels des institutions partenaires. Parallèlement à la demande de restitution par la Tanzanie du squelette du Brachiosaure du Pergamon Museum de Berlin, I'Allemagne a également proposé la mise en place d'un programme de formation de paléontologistes tanzaniens. Il est important de souligner que ces processus doivent rester mutuels et équitables et que les formations sont conçues pour s'adresser aux deux parties. Les musées qui abritent ces objets ont autant à apprendre de leurs homologues exerçant dans les institutions des pays d'origine.

Par ailleurs, les musées dont les collections ont été constituées en contexte colonial sont confrontés à un faisceau de relations incluant la violence coloniale, l'exil, l'expérience collective de la séparation et de la perte. Édouard Glissant décrit ce "Chaos-monde » résultant du contexte colonial comme « le choc actuel de tant de cultures qui s'embrasent, se repoussent, disparaissent, subsistent pourtant, s'endorment ou se transforment ${ }^{39} \ldots$ » Du «Chaos-monde » naît " La Relation», carrefour des échanges et « somme finie de toutes les différences du monde ${ }^{40} »$. Les objets conservés dans les collections se situent au carrefour de ces relations et leur patrimonialisation entre en résonance avec la théorie de La Relation développée par Glissant. Leurs trajectoires, leurs biographies et leurs parcours, d'un lieu à un autre, sont intimement liés au contexte historique, aux guerres, annexions, conquêtes, occupations coloniales ou étrangères, déplacements de frontières, mais aussi " aux curiosités esthétiques, intérêts scientifiques, expédiations militaires, réseaux commerciaux ${ }^{41}$ » qui ont motivé leurs déplacements. Ces objets incarnent donc des nœuds de relations où s'entremêlent les différentes composantes de leur parcours biographique. La mise en œuvre du projet de muséologie participative au Världkulturmuseet de Göteborg, en 2003, où des Suédois d'origine éthiopienne avaient été invités à réinterpréter les collections collectées un siècle plus tôt en Éthiopie, avait permis de mettre en exergue l'analogie entre l'objet réceptacle d'un faisceau d'interactions complexes qui s'entrecroisent et le nœud de relations formé qu'il incarne ${ }^{42}$. Emblématiques de ces échanges forgés dans des contextes composites, ces collections imposent la mise en place de programmes de recherche sur leur provenance et participent ainsi de la démarche d'inscrire l'histoire des contacts dans les réflexions du musée ${ }^{43}$.

Ariane Perrin. En Corée, les chercheurs qui s'intéressent à ces questions prennent plutôt position en faveur des restitutions s'il est avéré que les objets sont sortis du pays illégalement. Depuis 2014, la Overseas Korean Cultural Heritage Foundation a mis en place, avec le concours de chercheurs de l'université d'Ewha à Séoul, un nouveau groupe d'investigation qui s'emploie à analyser les archives japonaises du musée du gouvernement colonial japonais (1915-1945) dans le but de comprendre les modalités de transfert des biens culturels coréens au Japon, exportation qui reste illicite pour les Coréens, et de poser les bases légales pour de futures demandes de restitution. 
Paula López Caballero. Au Mexique, aucune restitution n’a eu lieu, étant donné le fond nationaliste qui soutient le patrimoine archéologique et le principe d'égalité pour tous les citoyens. On peut cependant offrir deux éléments de réflexion pour se pencher sur les articulations entre les scientifiques, les agents d'intervention publics et le monde juridique.

En premier lieu, la démarche scientifique et l'intervention publique ne se sont pas articulées pour la première fois dans le cadre des controverses ou des contentieux. La constitution même des collections en est la preuve. Au Mexique, I'implication des experts archéologues et anthropologues date au moins des premières décennies du $X X^{e}$ siècle. Des experts comme Manuel Gamio ou Alfonso Caso ont été des figuresclefs de la découverte et de la conservation du patrimoine archéologique, et d'une importance capitale pour convaincre les différents gouvernements de l'importance de cette tâche. Le rôle de ces hommes-institutions, qui ont traversé toute la première moitié du XXe siècle, au-delà de la recherche scientifique, a aussi consisté à maintenir le patrimoine et la conservation à l'agenda gouvernemental. Selon Manuel Gamio, anthropologue et archéologue chargé de la dernière phase de la découverte du site de Teotihuacán entre 1912 et 1917, c'est " pour mieux gouverner » - pour mettre en œuvre une meilleure " gouvernance », dirait-on aujourd'hui - qu'il est important de soutenir l'archéologie et l'anthropologie, argument d'où la recherche en sciences sociales tire sa légitimité depuis cette époque et, dans une certaine mesure, jusqu'à aujourd'hui. Cette interpénétration entre politique publique et recherche scientifique a, au Mexique, déterminé le développement de ces disciplines suivant une double dynamique. Tant l'anthropologie que l'archéologie ont trouvé une niche dans les institutions gouvernementales chargées de la conservation du patrimoine et de l'intégration des populations autochtones. Ces espaces institutionnels ont fourni aux scientifiques des budgets, et des lieux d'intervention concrets permettant d'alimenter la recherche. En contrepartie, la recherche a été, pendant presque tout le $x x^{\mathrm{e}}$ siècle, instrumentalisée par ces agences d'intervention publiques en fixant, jusqu'à un certain point, des lignes directrices générales à propos de ce qui devait être étudié et à quelles fins, comme la gloire nationale par l'archéologie, ou la consolidation d'une communauté nationale homogène où la « diversité culturelle » peut être exhibée comme une partie intégrante de la richesse du pays. Ces discours publics et la recherche qui leur était associée « explosent » à partir des années 1970, bien que des voix critiques se soient toujours fait entendre à distance de ce programme.

Si cette première configuration étatique et scientifique peut être appelée « nationaliste », celle qui domine aujourd'hui est généralement dite «multiculturelle ». Dans ce cadre, l'articulation de la recherche et de l'intervention politique a lieu, de plus en plus, dans la sphère juridique. Depuis les années 2000, en effet, certains droits spécifiques sont octroyés à des populations identifiées comme autochtones, notamment autour des questions électorales (procédures coutumières d'élection de représentants) et territoriales (consultations publiques sur les interventions privées et publiques sur leur territoire). Or, un enjeu majeur de ces procédures est de démontrer I'« indigénéité » des sujets afin qu'ils puissent formuler leurs demandes sur la base des « droits autochtones ». Le rôle des anthropologues est clef ici, car ce sont eux qui doivent émettre des rapports extrêmement solides justifiant cette forme d'identification. II n'est pas rare que dans ces situations, ils se sentent obligés de simplifier leurs résultats, par exemple en exaltant des continuités culturelles, en effaçant des conflits internes à la localité, voire en réifiant la culture locale pour répondre aux attentes des juges et des demandeurs. Un débat est né à propos de la validité de cet "essentialisme stratégique », dont le but est de soutenir des acteurs historiquement subordonnés ou marginalisés. Conséquence imprévue de cette interpellation de l'anthropologie par le droit, cette position $d^{\prime}$ « essentialisme stratégique » commence à avoir des effets sur la production scientifique 
elle-même. En effet, suite à l'élaboration de ces rapports, les anthropologues se sentent parfois moralement obligés de ratifier dans leurs publications scientifiques ce qui a été dit dans le rapport juridique. Face à cette situation, I'enjeu est donc de parvenir à fournir des arguments juridiques sans pour autant réifier les identités et les cultures. Ou, comme l'écrit Karen Engle ${ }^{44}$ de ne pas renoncer à la critique culturelle devant la loi, dans un effort pour que les exigences du système juridique vis-à-vis des populations cessent de reproduire des représentations essentialistes et réifiées de leurs formes de vie.

Ces réflexions peuvent à première vue sembler déconnectées des problématiques entourant les collections «non occidentales ». II me semble, cependant, que leurs enjeux n'en sont pas si éloignés, à condition de concevoir le problème non pas comme une question d'objets en circulation ( $\mathrm{I}^{\prime}$ « œuvre d'art non occidentale ») mais comme une question de rapports

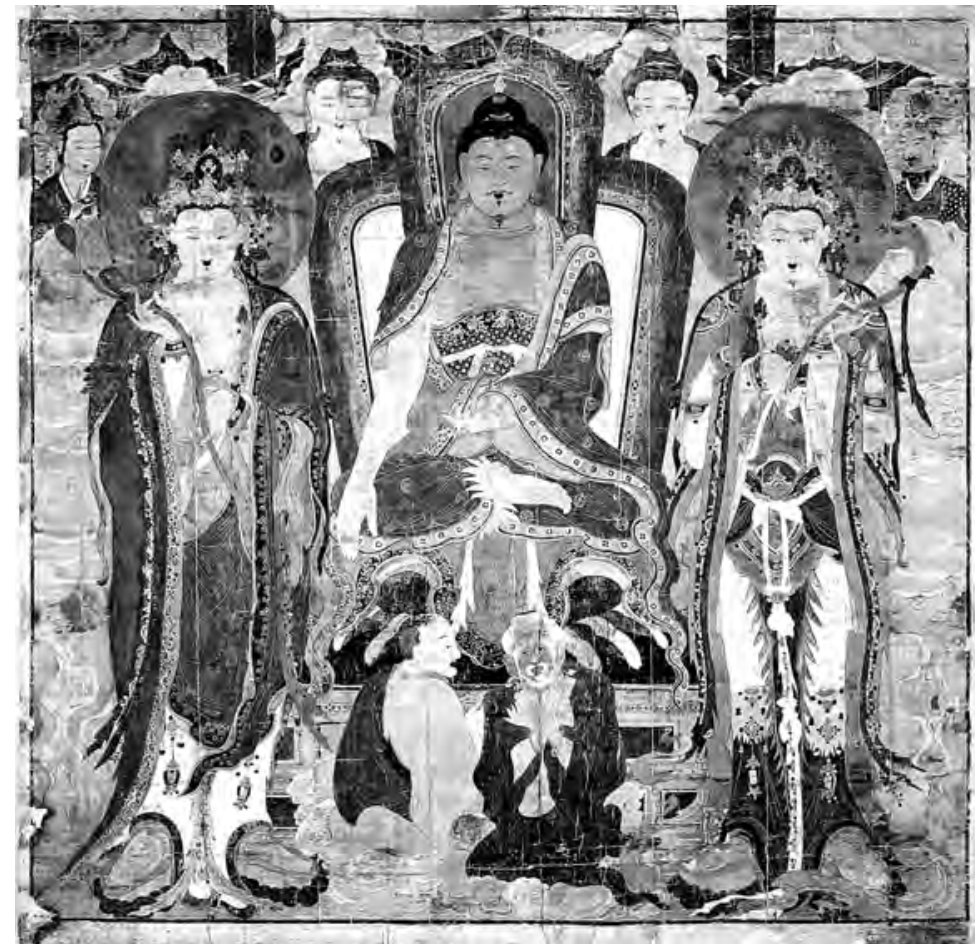
produisant des identifications, des différenciations et des hiérarchies. En effet, la première question à se poser est la suivante : quel type de sujets est-on en train de produire à travers les demandes de restitution ? Concoure-t-on, à travers les procédures de demande de restitution (tout comme les procès juridiques sur la base des droits autochtones), à une réification accrue de l'altérité du groupe, à une réactivation des fétiches de la modernité ? Cherche-t-on à creuser encore plus la frontière qui sépare des mondes qui se sont longtemps et intensément croisés, comme le fait la distinction « non occidentale »? Sans nier que, comme cela a déjà été dit, la réification d'une identité ou d'une culture puisse être utile à des fins d'obtention ou de protection des ressources, le travail du scientifique ne devrait-il pas être orienté aux fins de contourner ce langage essentialisant, sans pour autant renoncer aux demandes légitimes qui le mobilisent?

Claire Bosc-Tiessé. L'aspect économique apparaît peu dans les débats. Est-ce qu'il s'efface au regard des enjeux identitaires et idéologiques, ou est-il occulté pour d'autres raisons?

Ariane Perrin. C'est un aspect auquel je me suis intéressée au cours de mes recherches car des sommes d'argent importantes ont été engagées dans certaines restitutions sans que I'on connaisse les modalités de ces transactions. Il est d'ailleurs difficile d'obtenir des informations précises à ce sujet en dehors d'éléments fournis lors des conférences de presse organisées en Corée à l'occasion du retour d'un bien culturel. C'est donc un sujet en cours de recherche.

La Overseas Korean Cultural Heritage Foundation de Séoul, est chargée de recenser et d'étudier les collections d'art coréen conservées à l'étranger dans la perspective de faire rapatrier les biens culturels exportés illégalement. L'une des dernières restitutions obtenue par la Fondation est une peinture bouddhique du XVIII siècle, Triade 
de Shakyamuni (fig. 7), restituée à la Corée par le musée américain Hermitage Museum and Gardens (Norfolk, USA) en 2013. Cette restitution est intéressante à double titre : si officiellement elle a eu lieu sous l'égide de la Fondation, I'opération a été financée par un autre acteur du projet, la société américaine de jeux vidéo Riot Games Korea, qui a offert une compensation financière importante de 250000 dollars US au musée américain. En effet, Riot Games Korea collabore avec l'Administration du patrimoine culturel coréen depuis 2012 pour créer une base de données numérique sur les bâtiments historiques confucéens de la période Chosŏn. Ceci étant dit, la Fondation des biens culturels coréens à l'étranger et l'ordre bouddhique du Chogye disposent de fonds propres consacrés au rachat de biens culturels, notamment lors de ventes aux enchères.

Viola König. Cette question est importante. Les débats publics sont dominés par les historiens, les historiens de l'art, les spécialistes de politique culturelle et les journalistes culturels. Les questions d'ordre économique ne sont pas au centre de leurs préoccupations. Elles entrent seulement en ligne de compte lorsque ces objets ou ces œuvres d'art prennent une valeur monétaire reconnue internationalement (tout comme le montant de la valeur de leur assurance) ou lorsqu'il est question de fouilles ou d'exportations illégales. Les célèbres objets en bronze du Bénin qui se trouvent dans de nombreux musées européens illustrent le conflit entre acquisition légale (achat) conforme au droit européen et les problèmes éthiques et moraux que posent ces acquisitions. Bien que ces bronzes du Bénin aient été vendus aux enchères à Londres, leur histoire originelle comporte des irrégularités juridiques, et les acquéreurs savaient pertinemment que les Britanniques se les étaient appropriés par la force lors de l'expédition punitive britannique de 1897 après la destruction du palais du roi.

Dans leur société d'origine, la majorité de ces objets ethnologiques a une valeur culturelle. Les questions économiques ont également leur importance lorsque des demandes de compensation sont adressées aux États dans lesquels se trouvent les musées qui hébergent ces collections. La crainte de devoir s'acquitter de réparations élevées a longtemps poussé le gouvernement fédéral allemand à ne pas reconnaître les crimes perpétrés par les autorités allemandes dans les anciennes colonies du Reich, en Tanzanie par exemple. À cet égard, les propos du président français Macron ont également fait bouger les choses du côté des responsables allemands ${ }^{45}$.

Benoît de L'Estoile. La dimension économique est particulièrement importante, dans la mesure où les prix du marché de l'art conditionnent non seulement les possibilités d'acquisition (rendant par exemple quasiment impossible l'acquisition sur le marché de reliquaires kota par les musées gabonais), mais aussi les conditions de circulation des objets, par le biais notamment des valeurs d'assurance. Plus la « valeur » d'un objet augmente sur le marché, plus il devient coûteux de le faire circuler. Dans la configuration actuelle, les prix considérables atteints par certains objets, en salle des ventes par exemple, ont donc essentiellement des conséquences négatives pour les pays ou les groupes d'origine. De même, la mise en place d'espaces en mesure d'accueillir les objets dans des conditions de sécurité et de protection satisfaisantes implique un investissement matériel et humain considérable.

$C^{\prime}$ est parce que cette dimension matérielle est essentielle qu'il faut inventer des ressources nouvelles allant dans le sens d'une réduction des asymétries. Ainsi, on pourrait imaginer des formes de prélèvement sur les ventes en maisons d'enchère. Un pourcentage, même faible ( $2 \%$ par exemple), du produit de la vente ${ }^{46}$, sur le modèle du "droit de suite » bénéficiant aux créateurs dont les œuvres passent en vente publique, pourrait aller aux pays ou aux communautés d'origine des objets, afin de financer la mise en place de musées ou l'acquisition d'œuvres. 
Laurella Rinçon. Le Royal British Columbia Museum a annoncé, il y a quelques semaines, la création d'un fonds pour permettre aux peuples autochtones du Canada de financer les frais inhérents à leur participation aux programmes de restitutions. Cette initiative rappelle ce que les expériences précédentes ont démontré, à savoir la nécessité de mobiliser des financements importants pour faire aboutir les projets de retour des œuvres dans les lieux où elles ont été créées.

L'obélisque d'Axoum, emporté par Mussolini à Rome en 1937, en est un des exemples les plus célèbres. La restitution de la stèle, d'abord prévue par les accords d'armistice de 1947, a eu lieu quelque soixante ans après, en 2005. Le transport de Rome à Axoum, dans le cadre de l'opération « Restitution » a coûté quelque 6 millions d'euros, entièrement pris en charge par Rome. L'obélisque a été rapporté en Éthiopie en avril 2005, et sa réinstallation a donné lieu à des travaux de longue haleine qui ont permis l'érection de la stèle, le 4 juin 2008. Que ce soient l'impact de la valeur des biens sur le marché de l'art, les frais provoqués par leurs transports et leur assurance, ou le développement des infrastructures nécessaires pour assurer leur préservation et leur sécurité, le contexte économique est un des facteurs inhérents à la circulation des biens culturels, et qui se révèle souvent constituer un frein ou un ralentisseur.

De même, la restitution, le partage et la circulation des biens culturels peuvent être perçus par les pays d'origine comme un outil de développement socio-économique. Il s'agit pour eux d'augmenter le potentiel d'attractivité et d'investissement grâce au développement des secteurs culturel et touristique, facteurs de création d'emplois et de richesses et moyens de lutte contre la pauvreté. Dans cette dynamique le Bénin a entrepris de construire trois musées pour accueillir son patrimoine dispersé et « révéler au monde, dans un esprit nouveau, sa contribution et son rôle dans l'évolution de l'humanitét7 ».

Claire Bosc-Tiessé. Comment envisager aujourd'hui de continuer à constituer des collections et de quelles manières? Ne faut-il pas repenser complètement le modèle muséal et la circulation des œuvres? Comment penser des musées comme "universels" et comme "zones de contact " s'ils sont localisés seulement en Occident? Quelles pistes sont-elles ou pourraient-elles être explorées et quelle mise en cuvre serait possible?

La littérature ou d'autres formes de création pourraient-elles aider, au-delà de l'utopie ou grâce à l'utopie, à décaler les approches ? Par exemple, le petit livre d'Arno Bertina, Des lions comme des danseuses ${ }^{48}$, imagine un chef bamiléké demander la gratuité d'accès au musée du Quai Branly pour les Bamiléké, mettant en mouvement une lame de fond qui impose la libre circulation des auvres et/ou des personnes.

Laurella Rinçon. Au-delà de la restitution, la problématique aujourd'hui est celle de I'accès universel aux chefs-d'œuvre de l'humanité sur les autres continents, plus que celle de l'universalité des chefs-d'œuvre conservés dans les institutions occidentales. Peut-on parler d'un droit et d'une aspiration de tous, partout dans le monde, à la jouissance patrimoniale, et ce également dans les grandes capitales africaines?

La fameuse "Déclaration sur l'importance et la valeur des musées universels » plaide pour des œuvres mises à la disposition d'un public international et confirme le rôle des musées comme institutions « qui ne sont pas au service d'une seule Nation, mais des citoyens de chacune ${ }^{49} »$. Elle semble néanmoins se contredire en essayant de contourner la question des restitutions, car c'est un des moyens, pour les musées africains notamment, de constituer des collections et de fournir au public, aussi bien local qu'international, une offre patrimoniale comparable à celle des grands musées occidentaux, incidemment du groupe des dix-neuf signataires auto-proclamés « musées universels ». Comme le souligne le porfesseur George $\mathrm{Abungu}^{50}$, pourquoi les musées 


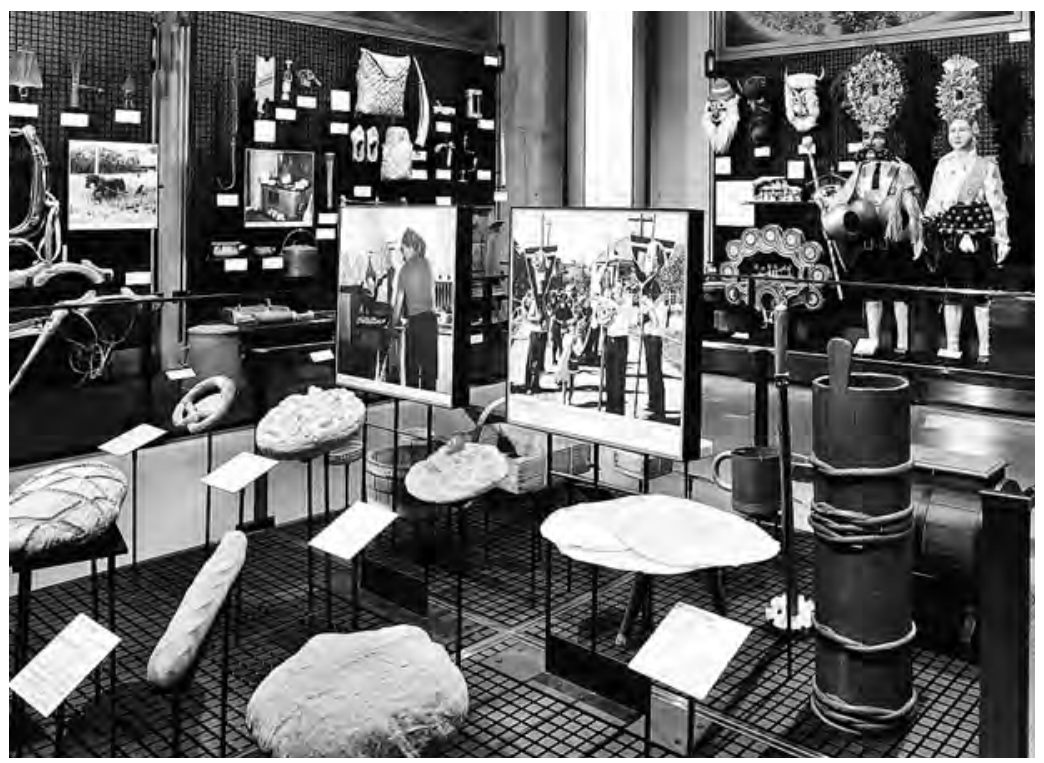

universels se trouveraient-ils uniquement en Europe et en Amérique du Nord et pourquoi seul ce public aurait un droit d'accès aux chefsd'œuvre de I'humanité ? II plaide notamment pour une évolution des pratiques professionnelles, et pour une plus grande prise en compte de la dimension immatérielle des objets qui complexifie les modalités d'appropriation du patrimoine. Ces œuvres d'importance majeure enrichissent ainsi le patrimoine national dans les pays qui les conservent, et s'inscrivent dans une ambition globalisée de diffusion du savoir et de la connaissance. En insistant sur le rôle des musées - favoriser une meilleure compréhension des différentes civilisations et promouvoir le respect entre

8. Vue de la salle consacrée aux cultures européennes, et en particulier à l'agriculture et au cycle annuel, Osaka (Japon), National Museum of Ethnology (Minpaku) elles -, les dix-neuf signataires plaident en réalité à leur insu, non seulement pour la restitution de biens culturels dans les sociétés où ils ont été créés, et où ils continuent de revêtir une forte signification cultuelle et culturelle, mais également pour une plus large mobilité des biens culturels qui permette de découvrir à Dakar, à Cotonou, à Nairobi ou à Addis Abeba, l'ensemble des cultures du monde, de la préhistoire à nos jours. C'est en partie l'ambition du Louvre Abou Dhabi et de la politique internationale lancée par le musée du Louvre.

Gageons que dans les années à venir, la politique d'éducation artistique et culturelle lancée par le Rijksmuseum, avec l'ambition que chaque jeune Néerlandais de huit ans ait visité au moins une fois le musée et ait été initié à ses vastes collections, soit réalisable au Musée des civilisations noires de Dakar, comme cela pourrait déjà être le cas aux musées nationaux du Kenya, ou à l'Iziko Museum du Cap.

Benoît de L'Estoile. Ces objets ont été rassemblés dans les musées d'ethnographie en fonction du paradigme scientifique de la collecte naturaliste, visant à réaliser une encyclopédie des cultures du monde. Le musée a été historiquement I'un des principaux lieux d'appropriation des « objets des Autres » par les Occidentaux; de quelle façon peut-il aujourd'hui participer au processus de réappropriation de la part des descendants de ceux auxquels ils ont été prélevés ou enlevés ? En d'autres termes, dans quelles conditions ce qui a été aliéné (de façon volontaire ou non) dans le passé peut, le cas échéant, être le support de réappropriations, y compris sous des modalités pour nous inattendues, avec des formes d'identification à certains éléments des collections, tels que des objets, des restes humains ou des photographies?

Cette nouvelle vie des objets entraîne pour les musées de nouvelles responsabilités, et de nouveaux défis. De façon minimale, on pourrait évoquer une exigence de « traçabilité », pour emprunter un terme aujourd'hui courant dans le domaine des produits alimentaires : c'est-à-dire un engagement du musée à rechercher l'histoire des objets qui figurent dans ses collections, et à la rendre publique. Il n'y a d'ailleurs pas de raison de limiter cette exigence aux seuls musées des autres : bien des collections de musée ont été le résultat non seulement de dons ou d'acquisitions, mais aussi de confiscations, de conquêtes ou de prélèvements forcés. Un tel effort de traçabilité 
favoriserait les possibilités de réappropriations multiples par les divers acteurs qui sont impliqués, tant du côté des publics, eux-mêmes diversifiés, que du côté des groupes d'origine et de ceux qui s'identifient à ces objets ${ }^{51}$.

Ainsi, la constitution de collections s'inscrit désormais dans une autre logique : il ne s'agit plus de constituer ou d'augmenter un " patrimoine national », mais plutôt de contribuer à mettre en place des dispositifs permettant la mise en place d'un « patrimoine partagé ». L'un des enjeux est précisément d'inventer de nouvelles modalités de coopération entre les musées du Sud et du Nord, fondées sur le principe de la symétrie. On peut s'inspirer du Minpaku, le Musée national d'ethnologie du Japon, à Osaka ${ }^{52}$. Celui-ci offre au visiteur européen une expérience unique : celle de se voir muséographié sous la forme de «l'autre » (fig. 8) : le musée expose des verres, des fourchettes, des cuillers, des baguettes de pain, un résumé de la culture paysanne française (avec notamment des objets du Berry). Ce musée a été à l'origine d'expositions mettant en scène le regard japonais sur les Occidentaux et le regard européen sur les Japonais, depuis les premiers contacts au XVle siècle. Ce cas suggère la possibilité de réaliser des expositions reposant sur le principe de la réciprocité et de la symétrisation des regards. Ainsi, on peut imaginer d'autres expositions qui problématiseraient la question du regard sur les objets des autres en insistant sur une approche de muséographie à la fois participative et symétrique. Il est également important de penser les expositions comme itinérantes ; idéalement, toute exposition organisée dans un musée occidental devrait avoir une forme de contrepartie dans le pays d'origine des œuvres.

Comment par exemple concilier les souhaits d'actuels habitants des îles Vanuatu de se réapproprier un objet rapporté en Europe par un missionnaire ou un marin, et donné à un musée, avec celui de la famille du donateur en faveur de la préservation de la mémoire du geste de son ancêtre ? Peut-on réconcilier appel à l'universel (qui est généralement associé à la revendication d'une identité cosmopolite) et revendication identitaire sur une base territoriale et généalogique ? Ces questions apparaissent insolubles si on les pense dans les termes juridiques habituels. Elles sont difficiles à penser dans le cadre d'un modèle juridique où le détenteur actuel qu'est le musée, au nom de la nation, serait pleinement propriétaire de façon absolue et irrévocable, comme l'implique la notion d'inaliénabilité : celle-ci annule l'histoire ou la transforme en « fait acquis » irréversible. Pour ouvrir de nouvelles façons de penser les questions d'appropriation et de réappropriation, il est donc nécessaire d'envisager le recours à d'autres schémas juridiques. L'enjeu est d'imaginer la possibilité d'une appropriation non plus sur le mode de l'exclusivité, mais sur un mode partagé. En d'autres termes, il s'agit de penser de façon prioritaire la question sur le mode de droits d'usages, et d'appropriations multiples, plutôt qu'à partir des modèles juridiques de la propriété et de la souveraineté.

D'autres modèles juridiques sont envisageables. Dans son Essai sur le don (1924), Marcel Mauss s'inspire notamment des descriptions de la Kula des îles Trobriand par Bronisław Malinowski pour expliquer que les bracelets prennent de la valeur à mesure qu'ils circulent, en fonction de leur parcours et de leur histoire. Mauss attaque la distinction occidentale entre le droit des personnes et le droit des choses comme une construction historique singulière. II indique que dans d'autres systèmes juridiques, par exemple chez les Maoris, la notion de hau induit que quelque chose de son propriétaire passé demeure attaché à l'objet, même quand il est donné. Dans cette conception, l'aliénation d'un objet ne rompt pas totalement les liens entre cet objet et ses origines. Mauss rapproche cela des discussions contemporaines autour du droit d'auteur. Précisément, I'auteur conserve des droits sur son œuvre dans des situations où il n'en est plus juridiquement propriétaire. L'acquéreur d'une œuvre d'art, par exemple un musée, ne peut l'utiliser absolument comme il l'entend : par exemple, si une œuvre est accrochée à l'envers, le créateur peut évoquer son droit 
moral d'auteur. Si l'on considère qu'une " communauté » peut avoir le statut de créateur d'une œuvre (comme le supposent les attributions ethno-géographiques), on peut lui attribuer une forme de droit moral sur celle-ci, et donc la possibilité d'une négociation sur les conditions de conservation, d'exposition ou de circulation des œuvres, par exemple. Ainsi, la créativité juridique est également en jeu dans le sujet que nous évoquons aujourd'hui, puisqu'il s'agit de réfléchir aux façons de rendre possible des formes d'appropriations plurielles.

Vincent Négri. La notion de musée universel a manifestement le vent en poupe, depuis 2002. Un vent qui souffle toujours depuis l'Occident. La référence à l'universalisme est séduisante mais, au regard du contexte dans lequel émerge la notion, il est loisible de s'interroger : la notion de musée universel n'est-elle pas une interprétation analgésique du musée prédateur? Si nous augmentons la focale, et resituons la notion dans le contexte plus large du droit international du patrimoine culturel, le concept de musée universel peut être analysé comme un déni des principes que la communauté internationale a développé, d'abord sous l'égide de l'Institut international de coopération intellectuelle de la Société des Nations, puis, à partir de 1945, sous les auspices de I'UNESCO. Rappelons que lors de sa 34 esession, en 2007, la Conférence générale de I'UNESCO a souligné le caractère équivoque de la notion univoque de musée universel, en affirmant que " la notion d'accès universel aux biens culturels exposés dans certains musées présentant un caractère universel ne saurait primer sur la notion morale et juridique de propriété du bien culturel, et que l'accès virtuel aux biens culturels ne saurait se substituer à la jouissance de ces mêmes biens dans leur cadre original et authentique ${ }^{53}$ ». On pourrait aussi, si ce n'est inverser le sens du vent, tout au moins s'intéresser à d'autres sources normatives qui, du point de vue des États africains, fondent un principe de circulation ou de restitution - autrement dit, de proposer une inversion du regard : depuis I'Afrique, que dit le droit sur la circulation et la restitution des biens culturels? - et I'on s'apercevrait que depuis et en Afrique le droit est également mobilisé sur cette question. Il s'agirait ainsi de poser les linéaments d'une autre écriture des rapports juridiques entre les musées.

Viola König. En ce qui concerne l'Allemagne, les ministres réfléchissent à adopter la gratuité d'accès aux musées berlinois. Ce sujet est toutefois secondaire étant donné que les descendants des sociétés d'où proviennent ces objets ne vivent pas en Europe. Dans ce cas, il faudrait aussi prendre en charge leurs frais de transport et de voyage.

Oui, aujourd'hui j'élaborerais un autre concept qu'il y a quinze ans lorsque j'avais été associée à la conception du Humboldt Forum. Je n'élaborerais plus de concept d'exposition, mais plutôt un concept de présentation. Le contact quotidien avec les collections telles que les connaissent les conservateurs dans les musées, ainsi que la transmission d'un certain savoir à des visiteurs profanes ont des conséquences sur le terrain que l'on ne peut pas anticiper en consultant la littérature scientifique.

Les concepts $d^{\prime}$ " universalité » et de "zones de contact » sont désuets, il faut continuer à les développer, comme c'est le cas à de nombreux endroits, par exemple en Amérique du Nord et en Océanie. Il est question de la pluralisation des concepts, de la prise en compte d'espaces dans lesquels ces objets doivent être réintégrés ; c'est aussi une question d'espace et de temps tels qu'ils ne sont ni connus ni vécus en Europe. En d'autres termes, il s'agit de déterminer quels sont les acteurs qui ont le droit d'interpréter ces collections indigènes provenant d'Amérique, d'Asie, d'Afrique et d'Océanie.

Dans la pratique, la manière de présenter ces collections à l'opinion publique en Occident doit être radicalement repensée. Ces collections détiennent un potentiel permettant d'aborder avec transparence et esprit critique les histoires nationales, 
européenne et globale. Il est impensable de recourir à des concepts d'expositions qui reposeraient sur une mise en scène. Ces collections doivent au contraire être mises à profit pour illustrer le morcellement qu'elles représentent tout en laissant aux descendants des sociétés d'où elles proviennent la possibilité d'exprimer leurs éventuelles réserves quant à l'exhibition publique de ces ressources. J'ai exposé ces idées dans un article paru récemment dans un grand quotidien allemand. Les réactions des lecteurs sont mitigées et les responsables sont plutôt interdits ${ }^{54}$.

Claire Bosc-Tiessé. Les débats qui accompagnent la mise en place du Humboldt Forum à Berlin font écho à certains de ceux qui ont accompagné la mise en place du musée du quai Branly à Paris il y a une quinzaine d'années. L'expérience de la réalisation $d u$ musée du quai Branly et surtout des controverses qu'il a soulevé a-t-elle eu des impacts dans le développement du projet berlinois? Ou les deux projets se sont-ils développés selon leurs logiques propres sans interaction?

Viola König. L'inauguration du musée du quai Branly et les longs débats qu'il suscita remontent à quinze ou vingt ans. Depuis cette époque, les discussions ont évolué. La question de savoir à l'époque si le musée du quai Branly misait excessivement sur une présentation esthétique, c'est-à-dire sur la dimension artistique, n'a pas été au centre des discussions autour du Humboldt Forum ; il a été davantage question de l'antagonisme entre l'apparence extérieure (la reconstruction du château de Berlin ; fig. 9) et son contenu (le transfert des collections africaines, américaines, océaniennes et asiatiques du musée de Dahlem). La nécessité d'une recherche scientifique d'envergure en matière de provenance des œuvres d'art et des biens culturels est au centre des discussions autour du Humboldt Forum - même avant les propos remarqués de Macron dans les médias.

Benoît de L'Estoile. Je ne connais pas précisément le processus d'élaboration du projet du Humboldt Forum, mais la question de la façon dont sont négociés les héritages coloniaux des deux côtés du Rhin est passionnante ${ }^{55}$. Le musée du quai Branly a eu un impact important dans le monde des musées, de par le succès public qu'il a rencontré, qui contraste avec le faible nombre de visiteurs de nombre de musées ethnographiques, qui apparaissent souvent aujourd'hui moins attractifs que d'autres. Il a réussi, en l'espace d'une dizaine d'années, à occuper une place de référence. Par conséquent, les nouveaux projets européens en tiennent nécessairement compte. Le musée du quai Branly est longtemps resté imperméable aux discussions dans d'autres contextes nationaux ou internationaux, mettant en avant son succès en termes de visiteurs pour couper court à tout questionnement. À leur tour, les vifs débats autour du projet du Humboldt Forum peuvent avoir un effet rétroactif en France : c'est ce que suggère la nomination, aux côtés de l'intellectuel Sénégalais Felwine Sarr, de Bénédicte Savoy, qui avait démissionné avec fracas l'an dernier du comité de pilotage du Humboldt Forum.

Laurella Rinçon. L'ouverture du musée du quai Branly en 2006, comme I'inauguration prochaine du Humboldt Forum sont des expériences qu'il faut resituer dans un contexte plus large, celui de la refondation des musées d'ethnographie européens, qui connaissent, depuis 20 ans, de profonds bouleversements. Ce phénomène a été qualifié par Jean Jamin de « crise des musées d'ethnographie » en 1998, lorsqu'il soulevait la question suivante, de manière provocatrice : «Faut-il brûler les musées d'ethnographie ${ }^{56}$ ? »

De I'achèvement du plan de gestion des collections au Rijksmuseum voor Volkenkunde de Leyde (dit plan Delta, 1997) à la réouverture du Weltmuseum de Vienne (2017), vingt ans se sont donc écoulés qui ont vu naître et s'intensifier ces débats sur la crise du musée européen d'ethnographie, en quête de réponses et de stratégies 


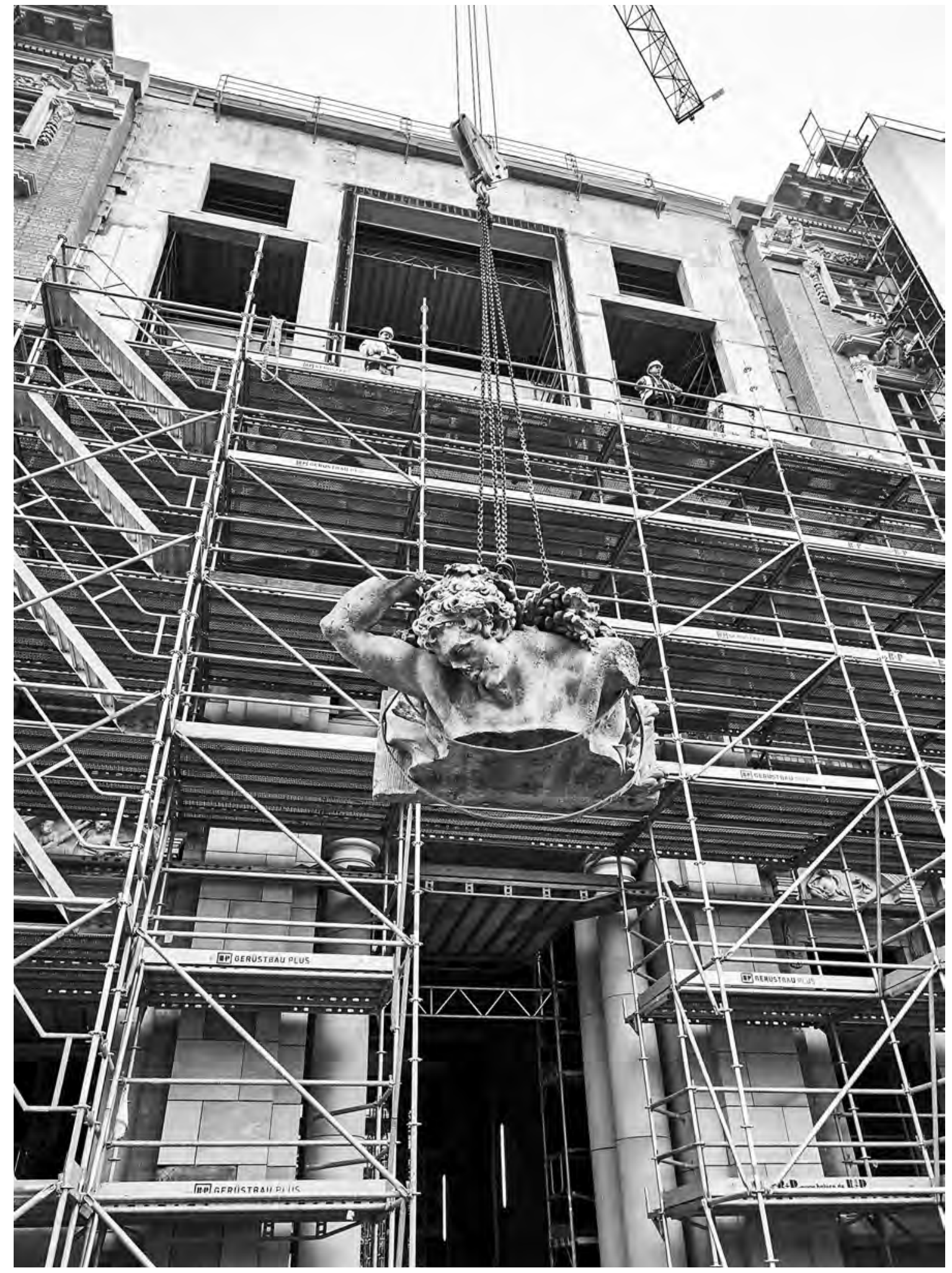


sur le devenir de ses collections. La liste des refondations est longue : la Sainsbury African Gallery du British Museum a ainsi réouvert en 2001, le Rijksmuseum voor Volkenkunde de Leyde en 2002, le Världkulturmuseet de Göteborg en 2004, le musée du quai Branly en 2006, les salles permanentes du Tropenmuseum, à Amsterdam, ont été rénovées en 2008 et 2009. Cette liste se prolonge dans une seconde vague, avec le Musée d'ethnographie de Genève en 2014, le Weltmuseum de Vienne en 2017, et sont à venir, parmi d'autres refondations, celle du Musée royal d'Afrique centrale de Tervuren en 2018, du Humboldt Forum de Berlin, du musée d'Ethnographie de Neuchâtel et du musée Pigorini à Rome.

Ces institutions s'influencent, travaillent en réseaux ${ }^{57}$, dans le cadre d'ateliers et de rencontres qui permettent de nourrir la réflexion et d'envisager selon un leitmotiv récurrent la "décolonisation du musée ». On constate tout de même que chacune de ces refondations reste profondément ancrée dans son histoire coloniale, dont aujourd'hui encore elle est un reflet saisissant. La question des restitutions joue dans ce contexte le rôle de catalyseur et accélère les processus de remise en cause d'une pratique muséale qui tarde à se renouveler en dépit des positionnements et des discours.

Claire Bosc-Tiessé. Dans une situation où les positions officielles semblaient verrouillées du côté des institutions étatiques européennes, le discours du président français, Emmanuel Macron, à Ouagadougou le 28 novembre 2017, crée une rupture. Des effets de cette déclaration se dessinent-ils?

Vincent Négri. Davantage qu'une rupture, le discours d'Emmanuel Macron décale sur un autre terrain la question de la restitution des biens culturels transférés en métropole pendant la période coloniale. Ce n'est plus l'argument de la légalité, armé par le double ressort d'une appropriation, avant qu'elle ne soit prohibée par le droit international, et de la domanialité publique de ces collections, qui fonde le discours officiel ; à cette légalité, le président de la République associe l'argument de la légitimité. On aurait tort de considérer que cette parole politique est nouvelle. Il y a 40 ans, le directeur général de I'UNESCO, Amadou-Mahtar M’Bow, publiait un appel «Pour le retour à ceux qui I'ont créé d'un patrimoine culturel irremplaçable » et, quatre ans plus tôt, en 1974, la Conférence générale de I'UNESCO adoptait une résolution mentionnant " la perte de biens culturels due à la colonisation et à l'occupation étrangère » et " déplor[ant] les transferts massifs d'objets d'art d'un pays à un autre, consécutifs à I'occupation coloniale ou étrangère ». Emmanuel Macron réactive une question lancinante à laquelle l'argument classique - occidental - de la légalité de l'appropriation et de la domanialité publique ne peut répondre durablement. En inféodant cet argument à celui de la légitimité, il pose les ferments pour inventer une autre légalité, armée par l'équité. Rappelons qu'en 1978 Amadou-Mahtar M’Bow posait le périmètre de ce droit au retour : "Aussi bien ces hommes et ces femmes démunis demandent-ils que leur soient restitués au moins les trésors d'art les plus représentatifs de leur culture, ceux auxquels ils attachent le plus d'importance, ceux dont l'absence leur est psychologiquement le plus intolérable. Cette revendication est légitime. "Sur cette question-là, le droit peut innover, en s'inspirant d'autres modèles ou en les inventant - en faisant œuvre de création juridique.

Benoît de L'Estoile. Il est trop tôt pour évaluer la traduction de cette déclaration dans des dispositifs concrets. En revanche, une telle déclaration a eu pour effet de « libérer des possibles ", en ouvrant une brèche dans le discours très fermé des musées occidentaux, français au premier chef, qui excluait presque systématiquement d'imaginer cette
9. Réinstallation d'un terme atlante de la façade du château baroque, porte 5 , pendant les travaux, Berlin, Humboldt Forum. 
hypothèse. La question est de savoir dans quelle mesure cette « déclaration d'intention » sera suivie d'effets, notamment du point de vue de la mise en place des conditions matérielles de réception des œuvres dans les pays africains, ou plus largement dans les pays dits du «Sud global », qui conditionne la possibilité de retours temporaires ou définitifs. On peut noter à ce propos que dans des pays comme le Mexique, qui ont mis en place, dès les années 1930, des politiques de protection du patrimoine national, les musées sont parfaitement équipés, et conservent des collections beaucoup plus riches que celles que l'on trouve hors du Mexique.

Dès à présent, on constate des effets dans le monde des responsables de musées et des conservateurs. Ainsi, I'anthropologue Nicholas Thomas, directeur du Museum of Archaeology and Anthropology de I'université de Cambridge, souligne la nécessité de prendre en compte les conditions matérielles de mise en œuvre de cette proposition, que « les installations de musée [en Afrique] doivent être améliorées, et qu'il faut renforcer les compétences en conservation et en muséographie. Il affirme que, dans nombre de cas, "le prêt d'œuvres à long terme rendra possible une conservation et une recherche conjointes, et peut être réalisé de façon plus rapide qu'une "restitution" complète $^{58}$ ». II appelle la Grande-Bretagne à s'inspirer de cet exemple. De même, Nanette Snoep, aujourd'hui directrice des musées d'ethnologie de Leipzig et Dresde, qui fut pendant plusieurs années en poste au musée du quai Branly, dans un texte appelant à « en finir avec le système de la colonialité59 », affirmait que la déclaration d'Emmanuel Macron constituait un « tournant décisif dans le débat sur le rapport à I'héritage colonial ». Elle évoquait ainsi son espoir de faire émerger un " musée postOuagadougou ». D'autres voix, venues d'Afrique, questionnent les motivations de ce geste, suspectant un cadeau symbolique pour faire oublier des inégalités structurelles. Au total, la déclaration d'Emmanuel Macron apparaît donc comme ayant " donné un coup de pied dans la fourmilière », ouvrant ainsi un nouvel espace des possibles.

Viola König. Cette bribe de discours devant des étudiants africains ne peut pas être isolée du contexte général du voyage en Afrique et de la nouvelle politique africaine de Macron. De par ses expériences de banquier et d'expert politique en économie et en finances, il utilise habilement le discours mainstream européen favorable à des restitutions pour mener sa politique et mise, pour ce faire, sur la carte culturelle. Les défenseurs européens d'une politique de restitutions et plus encore les partenaires africains en personne doivent désormais se montrer insistants et exiger sans faiblir la mise en œuvre de la proposition de Macron. Ce discours aura véritablement engendré une « rupture » seulement si des actes concrets s'ensuivent immédiatement, c'est-àdire si la France œuvre à la négociation et à l'organisation de restitutions d'objets provenant de sociétés africaines. Ces mesures ne doivent pas rester limitées à quelques cas exceptionnels qui serviraient de façade. Le musée, en tant qu'institution, est une invention européenne. Que la France construise de nouveaux musées en hommage aux sociétés africaines en vue de concilier ses intérêts culturels et économiques, est-ce vraiment ce à quoi aspirent en priorité les sociétés africaines ? Nos partenaires africains doivent exprimer clairement leurs souhaits, en se plaçant sur un pied d'égalité, et les faire respecter!

Lors de ma prise de fonction au Musée ethnologique en 2001, l'idée de restitutions était encore taboue; on n'avait par exemple pas le droit de parler de « pillages de sépultures » même si la provenance des objets était évidente. C'est pourquoi je me suis attelée, durant ces années à la tête du Musée ethnologique, à engager en douceur un début de politique de restitutions en initiant un changement de mentalités des responsables publics vis-à-vis des restitutions ainsi qu'en prenant des mesures préparatoires concrètes, toujours en collaboration très étroite avec les sociétés respectives 
d'où provenaient les objets concernés. Cela a porté ses fruits. J'ai fait des expériences très différentes en la matière. Le Musée ethnologique de Berlin, qui s'est montré prêt à restituer deux momies d'enfants provenant des îles du détroit de Torrès - opération pour laquelle une simple demande écrite aurait suffit - n'a pas obtenu de réponse depuis 2014, bien que l'ambassade d'Australie eût entamé précédemment des démarches dans ce sens. En revanche, l'ensemble du processus visant à restituer une collection qu'Adrian Jacobsen s'était appropriée en pillant des sépultures au cours d'une expédition en Alaska en 1881-1883 s'est déroulée en un laps de temps très court. La Chugach Alaska Corporation a déposé une demande de restitution en février 2016 et obtenu une réponse favorable dès décembre 2017.

Laurella Rinçon. Les effets sont rapides et semblent exponentiels. Un débat qui s'enlisait et ne suscitait pas d'intérêt partagé a été ravivé, et les prises de positions et déclarations politiques des décideurs, émanant autant de pays d'origine des biens, que de pays les conservant dans leurs musées et centres de recherche, se sont multipliées au cours de ces six derniers mois. La presse s'est ainsi fait l'écho de ce qui peut apparaître comme une course de vitesse, où institutions et États rivalisent d'effets d'annonce.

Si dans les pays scandinaves la question des restitutions est traitée depuis plus d'une décennie, il semble que le discours de Ouagadougou ait décrispé les positions en France où la question demeurait un impensé dans les milieux professionnels, voire un tabou. Mais « I'effet-Macron » agit également dans d'autres pays européens, I'Allemagne, entre autres, qui avait amorcé une réflexion et des collaborations avec de nombreux pays renforce sa volonté politique et ses actions emblématiques, comme pour ne pas être prise de vitesse. La publication, en mai 2018, par l'association des musées allemands, d'un guide de bonnes pratiques pour la gestion des collections issues d'un contexte colonial $^{60}$, inscrit la volonté de la ministre de la Culture Monika Grütters de porter cette question sur la scène politique et de lancer une réflexion dans les institutions.

En écho, I'UNESCO a organisé la conférence internationale "Circulation des biens culturels et patrimoine en partage : quelles perspectives ? \le $1^{\text {er }}$ juin 2018, comme pour réactiver l'appel «Pour le retour, à ceux qui l'ont créé, d'un patrimoine culturel irremplaçable ", lancé dès le 7 juin 1978, par Mr. Amadou-Mahtar M’Bow, alors directeur général. Il y a 40 ans, cet appel encourageait déjà à un changement de paradigme avec la mise en place du Comité intergouvernemental pour la promotion du retour de biens culturels à leur pays d'origine ou de leur restitution en cas d'appropriation illégale (ICPRCP). Ce contexte engage les États à mettre en œuvre un dialogue renouvelé sur la question de la mobilité des biens culturels aujourd'hui conservés hors de leur substrat d'origine. Des projets de partenariats émergent, dans l'objectif de renforcer les liens historiques qui unissent les États et deviennent emblématiques de formes de coopérations revisitées à l'aune d'un nécessaire dialogue interculturel.

La contribution de Viola König a été traduite de l'allemand par Bérénice Zunino. 
1. Ezio Bassani, African Art and Artefacts in European Collections 1400-1800, Londres, British Museum Press, 2000.

2. On peut par exemple regarder le sommaire du $n^{\circ}$ XXX/1 de Museum, revue publiée par I'UNESCO, consacré en 1979 au retour et à la restitution de biens culturels.

3. Ece Velioglu, Anne Laure Bandle, Alessandro Chechi, Marc-André Renold, "Case Three Nok and Sokoto Sculptures - Nigeria and France ", sur la base de données ArThemis, Art-Law Centre, University of Geneva, décembre 2012 [en ligne, URL : http://unige.ch/art-adr (consulté le 24 mai 2018)].

4. Seth Médiateur Tuysabe, Justine Ferland, MarcAndré Renold, "Affaire Bélier malien - France, Jacques Chirac et Mali », sur la base de données ArThemis, Centre du droit de l'art, Université de Genève, décembre 2015 [en ligne, URL : http:// unige.ch/art-adr (consulté le 24 mai 2018)].

5. http://www.elysee.fr/declarations/article/ discours-du-president-de-la-republique-emmanuel-macron-a-I-universite-ouaga-i-professeurjoseph-ki-zerbo-a-ouagadougou/ (consulté le 18 décembre 2017). En mars 2018, le président de la République française a ensuite confié une mission de réflexion et de consultation sur les conditions de restitution à Bénédicte Savoy, professeur d'histoire de l'art à la Technische Universität de Berlin et au Collège de France, et à Felwine Sarr, professeur d'économie à I'Université Gaston Berger de Saint-Louis, dont les conclusions doivent être rendues en novembre de cette année.

6. En raison des missions qui leur ont été confiées, Bénédicte Savoy et Didier Houenoude n'ont pas pu participer à ce débat comme cela était initialement prévu.

7. Laurella Rinçon, "Visiteurs d'origine immigrée et réinterprétation des collections au Världkulturmuseet de Göteborg ", dans Culture \& Musées, $\mathrm{n}^{\circ}$ 6, 2005, p. 111-127.

8. Je me permets de renvoyer à mon article "L'oubli de I'héritage colonial ", dans Le moment $d u$ quai Branly, numéro thématique de Le débat, novembre 2007, n 147 , p. 91-99, où je proposais de distinguer " colonisation » au sens strict, domination juridique par un pays européen de territoires extérieurs et " rapport colonial » désignant l'ensemble de modalités de rapports asymétriques entre I'Europe et les autres continents, dans une période qui va du $X V^{e}$ au $X X I^{e}$ siècle, qui prend des formes très variées allant de l'appropriation forcée à l'échange mutuel.

9. C'est à ces questions qu'est consacré le séminaire collectif : "Réécrire le passé colonial : enjeux contemporains des collections de musée ", voir : http://sciences-sociales.ens.fr/Reecrire-lepasse-colonial-enjeux.html.
10. Thomas Biersteker, Cynthia Weber (dir.), State Sovereignty as Social Construct, Cambridge, Cambridge University Press, 1996.

11. Fernando Coronil, «Beyond Occidentalism: Toward Nonimperial Geohistorical Categories », dans Cultural Anthropology, vol. 11, n 1, 1996, p. 51-87.

12. "[D]ifference is turned into otherness », Coronil, 1996, cité n. 11, p. 73.

13. "[I]nterconnected peoples come to lead separate lives », Coronil, 1996, cité n. 11, p. 73.

14. Birgit Schäbler (dir.), Area Studies und die Welt. Weltregionen und neue Globalgeschichte, Mandelbaum Verlag, Vienne 2007, p. 37 ; Dietmar Rothermund, Ibidem, p. 213.

15. Arnaud Nanta, «L'organisation de l'archéologie antique en Corée coloniale (1902-1940) : du terrain aux musées coloniaux », dans Ebisu, n 52, 2015, p. 117-154, ici p. 133 [en ligne, URL : http://ebisu. revues.org/1635 (consulté le 23 mai 2018)].

16. Mais ce n'est pas toujours le cas : le travail d'Elizabeth Ferry, par exemple, porte sur la patrimonialisation d'une mine d'argent par ses travailleurs, voir Elizabeth Emma Ferry, Not Ours Alone. Patrimony, Value, and Collectivity in Contemporary Mexico, New York, Columbia University Press, 2005.

17. Paula López Caballero, «Which Heritage for which Heirs. Pre-Colonial Past and Colonial Legacy in the National History of Mexico ", dans Social Anthropology / Anthropologie Sociale, Special Issue, no 16-3, 2008, p. 329-345.

18. Haydée López Hernández, En busca del alma nacional. La construcción de la "cultura madre" en los estudios arqueológicos en México (1867-1942), thèse de doctorat en philosophie de la science, Université Nationale Autonome du Mexique (UNAM), Mexico, 2010.

19. Il s'agit de la Fondation des biens culturels coréens à l'étranger, créée en 2012, et placée sous la tutelle de la puissante Administration du patrimoine culturel (Cultural Heritage Administration). Le décompte des biens culturels coréens conservés au Japon date du $1^{\text {er }}$ avril 2018. Voir la liste des pays qui possèdent des biens culturels coréens : http://www.overseaschf.or.kr/site/homepage/menu/ viewMenu?menuid=002003002. En dix ans et depuis 2008, le chiffre a plus que doublé passant de 76143 à 172316 biens recensés (2018).

20. Voir par exemple le cas des vols de statues bouddhiques coréennes au Japon, évoqué plus loin.

21. Pour certains chercheurs coréens, tel Yi Sunja, les fouilles conduites durant la colonisation étaient toutes illégales car imposées du fait de la domination coloniale ; alors que les autorités japonaises opposaient fouilles légales conduites en cohérence avec leurs recherches sur I'histoire de la péninsule coréenne et pillages illégaux. Voir I'article d'Arnaud Nanta, "Les débats au XXe siècle 
sur la légalité de l'annexion de la Corée : histoire et légitimité ", dans Cipango, no 19, 2012, p. 75-110 [en ligne, URL : https://cipango.revues. org/1676 (consulté le 23 mais 2018)].

22. "Agreement on Art Objects and Cultural Cooperation ». La Corée avait soumis une liste de 1431 biens culturels coréens conservés au Japon (dont 544 antiquités et 852 ouvrages) en vue d'une restitution. Voir le texte du traité : https://treaties. un.org/doc/Publication/UNTS/Volume\%20583/ volume-583-I-8473-English.pdf.

23. « Déclaration sur l'importance et la valeur des musées universels ", décembre 2002, disponible sur le site internet du British Museum (en anglais) : www.thebritishmuseum.ac.uk/newsroom/current 2003/universalmuseums.htm.

24. Colloque " Les nouveaux visages de l'interculturalité. Quel patrimoine, quel musée, quelle bibliothèque, quel objet à I'heure de l'interculturalité et du métissage des populations et des cultures ? Pour quels publics?", Paris, Bibliothèque nationale de France, 18 novembre 2004.

25. George Abungu, "The Declaration: a Contested Issue », dans ICOM News, vol. 57, n 1, 2004.

26. Paracas: A Stolen World, Göteborg, Världkulturmuseet, 18 septembre 2008 - 9 janvier 2011.

27. Les forêts natales, arts d'Afrique équatoriale atlantique, Paris, musée du quai Branly, 3 octobre 2017 - 21 janvier 2018.

28. Barbara Cassin, Danièle Wozny (dir.), Les intraduisibles du patrimoine en Afrique subsaharienne, Paris, Démopolis, 2014.

29. Emmanuel Kasarhérou, Gaye Sculthorpe, Nicolas Thomas, Michel Coté et Vincent Négri, "Dialogue des cultures et circulations des œuvres ", dans Musée du quai Branly - Jacques Chirac, 10 ans après, actes du colloque (Paris, musée du quai Branly, 2016), Paris, musée du quai Branly (Les actes), 2017, [En ligne, URL : http://actesbranly.revues.org/794 (consulté le 12 novembre 2017)]. Voir aussi le catalogue de l'exposition: De jade et de nacre : patrimoine artistique kanak, cat. exp. (Nouméa, musée territorial de Nouvelle-Calédonie, 1990 / Paris, Musée national des arts africains et océaniens, 1990-1991), Paris, Réunion des musées nationaux, 1990.

30. Ce programme a malheureusement été interrompu depuis faute de financement, ce qui pointe une des principales difficultés rencontrées par les programmes d'exposition coopérative : celui du financement. Bien des programmes qui prévoient une coopération entre musées européens et africains s'arrêtent, ou se limitent au financement des voyageurs européens pour aller en Afrique.

31. Conférence internationale, "Circulation des biens culturels et patrimoine en partage : quelles

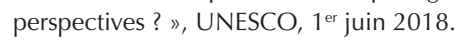

\section{Ibidem.}

33. II n'est que de citer les accords conclus entre I'Italie et les musées américains entre 2006 et 2008, concernant des biens archéologiques issus de pillages en Italie et acquis par ces musées américains dans les années 1970. Sur un autre versant, les modèles juridiques mis en œuvre pour le patrimoine naturel pourraient également façonner $d^{\prime}$ 'autres modes de penser les revendications; il ne s'agit plus d'envisager la question au seul prisme de la propriété, mais de reconnaitre les diversités de contextes et la pluralité des droits qui y sont attachés. Cet enjeu se joue aussi dans les appariements entre diversité culturelle et biodiversité (je me permets de renvoyer à la recherche collective, dont j'ai dirigé la publication : Vincent Négri (dir.), La diversité dans la gouvernance internationale. Perspectives culturelles, écologiques et juridiques, Bruxelles, Bruylant, 2016).

34. Voir le décret $n^{\circ} 2011-527$ du 16 mai portant publication de l'accord entre la France et la république de Corée relatif aux manuscrits royaux de la Dynastie Joseon, signé à Paris le 7 février 2011 (Journal officiel, 18 mai 2011).

35. Voir par exemple le catalogue : 145 nyŏn manŭi kwihwan, oegyujanggak ŭigwe / 145년 만의 귀환, 외규장 각 의궤 (The Return of the Oegyujanggak Uigwe from France: Records of the State Rites of the Joseon Dynasty), cat. exp. (Séoul, National Museum of Korea, 2011), Séoul, National Museum of Korea, 2011.

36. Manuel Buróno Díaz, "Comunidades, patrimonio y arqueólogos: relaciones entre municipios e instituciones culturales en Oaxaca en el periodo indigenista ", dans Estudios Ibero-Americanos, vol. 42 , no 1 , ene.-abr. 2017, p. 67-80.

37. Sandra Rozental, " Unearthing Patrimonio: Treasures and Collectivity in San Miguel Coatlinchan », dans Olaf Kaltmeier et Mario Rufer (dir.), Entangled Heritage: (Post)Colonial Perspectives on the Uses of the Past in Latin America, Londres, Ashgate/Routledge, 2017 ; Eadem, La piedra Ausente, long-métrage documentaire, co-dirigé avec Jesse Lerner, coproduction Institut national d'anthropologie et histoire (INAH) et Instituto Mexicain de Cinématographie (IMCINE), 2013 (voir : www.lapiedraausente.com).

38. Eduardo Abaroa, Destrucción total del Museo de antropología, Mexico, Athenée Press, 2017.

39. Édouard Glissant, Poétique de la Relation, Poétique III, Paris, Gallimard, 1990.

40. Édouard Glissant, La cohée du lamentin. Poétique V, Paris, Gallimard, 2005.

41. Bénédicte Savoy, "Restitution du patrimoine africain, il faut y aller dans la joie ", dans Le Monde du 12 janvier 2018.

42. La notion qui émerge à l'occasion de cette expérience est décrite dans I'article de Rinçon, 2005, cité n. 7, et a été développée au prisme 
des théories de La Relation d'Édouard Glissant, notamment dans I'article de Laurella Rinçon, " De Dakar au Havre, échos d'une muséographie de La Relation ", dans Hamady Bocoum, Étienne Féau, Cédric Crémière, Vers le musée africain du XXI ${ }^{e}$ siècle, Le Havre / Paris, Muséum du Havre / MKF éditions, 2018.

43. Laurella Rinçon, Le musée comme zone de contact, nouveaux discours, nouvelles pratiques, Paris, Alternatives, 2018.

44. Karen Engle, The Elusive Promise of Indigenous Development: Rights, Culture, Strategy, Durham, Duke University Press, 2010.

45. Arlette-Louise Ndakoze, "Koloniale Verantwortung Deutschlands. Das Erinnern an Kolonialismus-Verbrechen wirkt allmählich ", dans Deutschlandfunk Kultur, 28 mars 2018 [en ligne, URL : http://www.deutschlandfunkkultur.de/ koloniale-verantwortung-deutschlands-das-erinnern-an.1005.de.html?dram:article_id=414128].

46. Les frais de vente, à la charge du vendeur, sont couramment de $18 \%$ TTC du produit de la vente, auxquels peuvent s'ajouter des frais d'expert.

47. S. E. Patrice Talon, président du Bénin, allocution d'ouverture, conférence internationale, "Circulation des biens culturels et patrimoine en partage : quelles perspectives ? ", UNESCO,

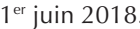

48. Arno Bertina, Des Lions comme des danseuses, Lille, La Contre-allée (Fictions d'Europe). Publié en 2015, le récit imagine une série d'événements qui se déroulent dans les années 2015-2019.

49. Talon, 2018, cité n. 47.

50. Talon, 2018, cité n. 47.

51. Il faut noter en ce sens l'effort que réalise le musée d'Ethnographie de Genève. En particulier, les objets présentés dans l'exposition permanente sont documentés sur un site très précieux. Voir par exemple : http://www.ville-ge.ch/meg/incontournables_section.php?section $=45$.

52. Voir le site du musée : http://www.minpaku. ac.jp/english/.

53. Résolution UNESCO $34 \mathrm{C} / 44$, adoptée le 2 novembre 2007, portant sur le renforcement de la protection des objets culturels par la lutte contre leur trafic illicite et le développement des musées dans les pays en développement.

54. Viola König, "Zeigt endlich alles! Warum nur ein radikales Konzept das Humboldt Forum noch retten kann. », dans Die Zeit, 28 avril 2018 [en ligne, URL : https://www.zeit.de/2018/18/ humboldt-forum-berlin-sammlung-gewalt-aufklaerung].

55. Avec un collectif de chercheurs, nous poursuivons un projet intitulé « France et Allemagne face aux héritages coloniaux : relectures contemporaines des collections de musée », voir : http://www.ciera. fr/fr/node/13842.

56. Jean Jamin, « Faut-il brûler les musées d'ethnographie? ", dans Gradhiva, n² 24, 1998, p. 65-70.

57. International Network of Ethnography Museums (RIME), Benin Dialogue Group, European network of diasporas associations and ethnographic museums (READ ME), Sharing a World of Inclusion, Creativity and Heritage (SWICH).

58. Nicholas Thomas, "We Need to Confront Uncomfortable Truths About European Colonial Appropriation ", dans The Art Newspaper, 27 novembre 2017 [en ligne, URL : https://www.theartnewspaper. com/comment/macron-repatriation].

59. Nanette Snoep, "Schluss mit dem „System der Kolonialität"! », dans Die Welt, 20 février 2018 [en ligne, URL : https://www.welt.de/print/die_welt/ kultur/article173757893/Schluss-mit-dem-Systemder-Kolonialitaet.html].

60. "Guide to Dealing With Collection Goods From Colonial Contexts" outline methodologies for provenance research and possibilities for restitution, Berlin, German Association of Museums, may 2018. 